\title{
THOMAS S. KUHN Y LA FUNCIÓN DE LOS INSTRUMENTOS CIENTÍFICOS EN EL DESARROLLO DE LAS CIENCIAS FISICAS
}

\author{
JuAn Vicente Mayoral de LuCAS
}

ABSTRACT: This is a study of Kuhn's ideas regarding the role of physical instruments in the historical development of physical sciences. Its approach to Kuhn's thought is primarily historical and biographical, and it makes extensive use of Kuhn's archival sources at MIT to settle a group of ideas of Kuhn's as a basis out of which that role follows almost naturally. It starts out with an introduction to the "operational" history of science Kuhn found as an assistant to Conant. It runs on talking about Kuhn's divergent opinions about the proper place of concepts in both physical research and theory. It ends with an account of Kuhn's new historical vision of science (including the role of instruments in it), and its relations to that of Alexandre Koyrés (another key influence on his thought).

\section{Introducción}

William Thomson (después Lord Kelvin) fue un defensor paradigmático de la medición precisa como recurso básico y necesario de la filosofía natural (lo que incluía la mecánica, la óptica, la teoría del calor, la electricidad y el magnetismo). Para él, la parte experimental de la filosoffa natural se ocupaba de dotar a la teoría de la sensibilidad y precisión necesarias mediante el uso de instrumental cientifico. Asimismo era un amante de la teoría matemática bien construida, y consideraba por ejemplo que la teoría de J. Fourier de la conducción del calor era «un gran poema matemático». Pero, a su modo de ver, la teoría del calor consistía en "examinar y apreciar la naturaleza de la temperatura" (cursivas mías) y esa "apreciación", que en este caso se lograba con un "termómetro sensible», era para él sinónimo de "medición". Así, no es extraño que aunque Fourier fuera para Thomson una suerte de genio creador, H.-V. Regnault, el gran físico experimental 
francés reconocido por sus investigaciones sobre el calor, fuese también para él un "maestro de la medición experimental».

Ahora bien, para Thomson la experimentación no era un recurso sólo de la filosofía natural. En la filosofia natural no tenía cabida la recopilación de datos que precedía al establecimiento inductivo de las leyes naturales, y ésta no dejaba de ser experimental. Había disciplinas como la geología o la fisiología vegetal y animal, cuyo método (y rasgo esencial) era la recogida y la clasificación de datos. Pero éstas eran historia no filosofía natural. Por lo tanto, puesto que había una parte de la experimentación física que se practicaba para la pura recogida y clasificación de datos, esta parte era para Thomson una fase de historia natural correspondiente a la filosofia natural ${ }^{2}$.

En todo ello nos encontramos con un claro problema. Si, como hemos visto, para Thomson la experimentación implica agudizar los perfiles cuantitativos de ciertos rasgos selectos de los fenómenos naturales, la naturaleza y función del experimento y la medición en la fase de historia natural se nos escapa. No sabemos cómo se "descubre» a la par que se «mide». Es decir, una vez que medimos, ¿̨cómo sabemos dónde nos conducen los resultados divergentes (i.e., si a la modificación de nuestros criterios de error aceptable o a la de las propias clasificaciones)? Y si, por otro lado, las clasificaciones se desarrollan y mejoran de las dos maneras (lo que parece la posición más aceptable), ¿̨cómo podemos vencer la tentación de incluir alguna forma de disposición psicológica (y, claro está, cualitativa) en la in-

1 Crosbie SMITH y M. Norton WISE, Energy \& Empire: A Biographical Study of Lord Kelvin, Cambridge: Cambridge University Press, 1989, esp. pp. 121-128. El último calificativo de Regnault es de los propios autores del libro. Cabe recordar que Jean-Baptiste-Joseph Fourier, en su Théorie analytique de la chaleur (1822), proponía un método matemático basado en ecuaciones diferenciales en derivadas parciales para describir los fenómenos del calor, tratado éste como un fluido y sin especificar ningún mecanismo de interacción mecánica. Por su parte, Henri-Victor Regnault intentó aumentar el grado de exactitud cuantitativa en diversas mediciones experimentales, desarrollando junto a otros físicos como R. Bunsen o H. Kopp nuevos instrumentos, y contribuyó a conocer mejor el comportamiento de los gases bajo presión. Thomson dominaba la teoría de Fourier a los quince años y estudió con Regnault en París a los veintiuno; de ahí sus comentarios. Sobre todo ello, cf. también, junto al texto antes citado, Crosbie SMITH, "Force, Energy, and Thermodynamics", en The Cambridge History of Science, vol. 5: The Modern Physical and Mathematical Sciences, Mary Jo Nye (ed.), Cambridge: Cambridge University Press, 2003, cap. 15 (pp. 289-310), esp. pp. 290-304; Mary Jo NYE, Before Big Science: The Pursuit of Modern Chemistry and Physics, 1800-1940, Cambridge (Mass.): Harvard University Press, 1999 (orig. 1996), pp. 48, 65-68 y 94-95.

${ }^{2}$ SMITH y WISE, op. cit., p. 122. 
tención y la práctica del historiador natural? Así, si hay diversas actitudes o disposiciones en la investigación tendrá que haber también más de una función para los instrumentos científicos.

El esquema con el que Thomson ordenó la filosofía natural a mediados del siglo XIX (del que sólo hemos ofrecido un burdo esbozo) era todavía válido al despertar el siglo XX, y en sus cuatro primeras décadas una filosofía del lenguaje y de la ciencia tan sofisticada y terapéutica como el positivismo lógico de Rudolf Carnap, Carl G. Hempel, etc., (sobre todo al principio de la misma) contemplaba ese escalonamiento de (1) clasificación pura de los datos de medición, (2) inducción teórica y (3) deducción de consecuencias para la contrastación empírica como el orden elemental sobre el que edificar la moderna teoría positiva del método. En algo tenían razón esos positivistas: la imagen del método que comunica la ciencia es paradigmática. Nuestra actitud pretendidamente racional se edifica en torno suyo. La historia de la ciencia que se extrae de ese patrón se convierte así en un registro de imágenes conforme a las cuales forjamos nuestra conducta, sobre todo si nos ocupamos de la investigación científica. Pero si existen las disposiciones psicológicas tanto en la recopilación como en la ordenación de los datos empíricos, esa historia de la ciencia bien podría cambiar y la propia imagen paradigmática de la actitud racional podría ser distinta.

A partir (más o menos) de la segunda mitad del siglo XX esta segunda posibilidad post-kelviniana empezó a tomarse en consideración. La filosoffa de la ciencia ha sido desde entonces mucho más consciente de las premisas metodológicas implícitas en la historia de la ciencia, y ha tratado de elaborarlas y de eliminar en lo posible las "contaminaciones" impropias de una imagen positiva del método científico y su historia. En este artículo vamos a hablar de uno de los artífices de ese punto de inflexión en la metodología de la ciencia, Thomas S. Kuhn. Nuestra excusa es la función de los instrumentos científicos, ya que éstos son símbolos tangibles de la imagen kelviniana. Kuhn los reubicó desde un punto de vista funcional de acuerdo con su revisión de la imagen de la historia de la ciencia y de la naturaleza del método, así que son un magnífico objetivo para pasar revista a la perspectiva pionera de Kuhn, a la par que averiguamos su nueva función en ella.

Nuestro artículo es histórico y biográfico. Expondremos las raices de la transición de Kuhn desde una visión "positivista" (e incluso operacionalista) del método científico (que no es la de los lógicos centroeuropeos) a su propia perspectiva. Mostraremos asimismo qué historia de la ciencia aprende y cómo la corrige a 
medida que se produce esa transición en sus ideas filosóficas. En lo referente a los protagonistas de esta historia, hablaremos de las influencias del análisis operacional de Percy W. Bridgman en la concepción de la historia de la ciencia y del método científico que Kuhn aprende al principio; veremos cómo James B. Conant le introduce a ella; examinaremos cómo ahonda Kuhn en sus nuevas ideas gracias a la obra de Alexandre Koyré; y finalmente veremos cómo Kuhn diverge tanto de Conant como de Koyré y dónde nos conduce esa divergencia. Al final lograremos comprender qué función poseen para Kuhn los instrumentos científicos, cómo (a pesar de Koyré) son un elemento esencial del desarrollo histórico de la ciencia, y cómo esa historia deja de ser con Kuhn la que llamamos «historia operacional» (más propia de Conant).

\section{La "historia operacional" de la física}

Thomas S. Kuhn no es un desconocido para la cultura del siglo XX. En general no lo es para la filosófica, pero mucho menos lo es en el ámbito de los estudios sobre la ciencia. Kuhn orientó a la filosofía de la ciencia hacia la historia de la ciencia a partir de los años sesenta del siglo pasado. El punto de partida de su transformación fue el conocido The Structure of Scientific Revolutions de 1962, un hito de la filosofía de la ciencia de la segunda mitad de siglo ${ }^{3}$.

En Structure, Kuhn proponía un papel para la historia de la ciencia como campo de trabajo para los filósofos de la ciencia. Su intención era mostrar una serie de problemas pendientes de resolver que se revelaban sólo a través de una profundización en el pasado de las ciencias que no fuera aquélla que los libros de texto de ciencias exhibían en sus introducciones o implicaban en las ordenaciones de sus teorías, métodos y conocimientos.

Tales problemas son los propios del desajuste entre la imagen filosófica de la ciencia y la imagen obtenida poco a poco por los historiadores. En la filosofía de la ciencia de los años treinta y cuarenta había una imagen más o menos clara de lo

3 Los promotores del enésimo intento de conciliar a sociblogos y a científicos (principalmente físicos) están, al menos, de acuerdo en este punto. Para bien o para mal, Kuhn proporcionó un impulso a los estudios sobre la ciencia que no ha dejado de preocupar a los que se ocupan de ello (continua o eventualmente) desde entonces. Cf. Jay A. LABINGER y Harry Coluins (eds.), The One Culture?: A Conversation about Science, Chicago: The University of Chicago Press, 2001, esp. «Introduction» (pp. 1-10), p. 4. 
que hacía de una empresa de investigación algo científico. Según esa imagen vaga, toda investigación científica tenía como fundamento metodológico organizar los fenómenos para proporcionar con ello una descripción y explicación de la realidad, pero sin tocar esos mismos fenómenos con las propias creencias y disposiciones. La observación pura era, por lo tanto, perfectamente posible. Más aún, era un objetivo metodológico claro. Los problemas de la filosofía de la ciencia eran los de hallar el algoritmo teórico adecuado para reducir esa imagen vaga a un solo grupo de principios metodológicos a los que ajustar toda teoría científica -y sólo las teorías científicas, es decir, con vistas a poder excluir de manera justificada toda pretendida empresa de investigación que no fuera en realidad más que una pseudo-ciencia-. La búsqueda de esos preceptos fue ardua. El desarrollo de la teoría metodológica chocaba con la abundante evidencia sobre la complejidad de lo que la ciencia hacía en realidad. Con todo, y a pesar de que el relato del desarrollo nos revela un trabajo de continuas correcciones a la teoría inicial, la mencionada imagen vaga imperaba tanto en la filosofía de la ciencia como en las concepciones del ciudadano de a pie $e^{4}$.

Kuhn hablaba de que los historiadores de la ciencia tenían, no obstante, muchas dificultades con esa imagen vaga, ya que veían difícil encontrarla cuando se asomaban a fases previas de la ciencia -incluso respetando que se trataba de una imagen sumamente vaga-. Galileo o Newton, por ejemplo, (y desde luego Aristóteles y Ptolomeo) encajaban sólo con dificultad y de manera muy artificial en el estereotipo filosófico de la ciencia. La observación pulcra de los fenómenos naturales, prototípica de la filosofía de la ciencia de esos años, caracterizaba grosso modo el ideal de la ciencia de cualquier periodo. A pesar de ello, su papel específico como germen único de toda investigación novedosa era muy dudoso; al menos del modo extremadamente sencillo en que lo presentaba la filosofía. La presencia

4 Para una muestra sintética y muy ilustrativa de esos esfuerzos, cf. Carl G. HEMPEL, "Problems and Changes in the Empiricist Criterion of Meaning", Revue internationale de philosophie, 4 (11) (1950), pp. 41-63. Versión revisada como capítulo IV de sus Aspects of Scientific Explanation, and Other Essays in the Philosophy of Science, Nueva York: Free Press, 1965, pp. 101-122. Este desarrollo puede verse también en el ya clásico texto de Frederick SUPPE (ed.), La estructura de las teortas cientificas, trad. Pilar Castrillo y Eloy Rada, Madrid: Editora Nacional, 1979 (orig. 1974), y esp. en la introducción histórica del autor a la conocida como "concepción heredada», pp. 21-266, y esp. pp. 21-85. El trabajo de Suppe proporciona muy pocos detalles historiográficos. Con ese objeto, cf. por ejemplo los diversos artículos compilados en Friedrich STADLER (ed.), Scientific Philosophy: Origins and Developments, Vienna Circle Institute Yearbook (1993), No 1, Dordrecht: Kluwer Academic Publishers, 1993. 
en los ejemplos históricos de otros motores alternativos a la "pura observación" ponía de manifiesto que quizá los filósofos tenían una imagen demasiado ingenua de la empresa científica. Structure tenía como objetivo generalizar esa complejidad de la imagen histórica para promover un cambio en el trabajo filosófico 5 .

Desde el punto de vista del historiador de la ciencia, esta crítica de Kuhn no era de modo alguno nueva en los años sesenta. En los años 1920-50, el belga (afincado en Harvard) George Sarton ya había puesto de manifiesto que la ciencia no avanzaba desde lo más fundamental a los elementos derivativos, con lo cual las nuevas teorías tendían a revisar los fundamentos de las previas una vez superadas éstas últimas. Las nuevas teorías, decía Sarton, fagocitan el contenido positivo de las segundas bajo una nueva estructura teórica más general ${ }^{6}$. Esto, para Sarton, revelaba el progreso mismo del espíritu humano pero también lo engañoso del orden de presentación del conocimiento científico en los manuales. Por ese motivo, el historiador de la ciencia era, en el estrato universitario, un servidor del conocimiento científico, el especialista que revelaba el orden de aparición de aquellos componentes de nuestra ciencia que las necesidades pedagógicas ayudaban a borrar. "No tomemos en consideración el argumento de la importancia de la ciencia como factor esencial de civilización" , decía Sarton, "(...) al ser la enseñanza de la ciencia inevitablemente no-histórica o anti-histórica, la historia de la ciencia se debe enseñar como complemento y corrección" ${ }^{7}$. Un historiador, alumno y amigo fiel de Sarton, como I. B. Cohen, decía algo muy parecido en los años cincuenta. En una conferencia para profesores de educación primaria y secundaria de febrero de 1950, Cohen advertía que el principal problema de los docentes

5 Thomas S. KuHn, The Structure of Scientific Revolutions, 3a ed. rev., Chicago: The University of Chicago Press, 1996, sec. I, esp. pp. 1-4.

${ }^{6}$ Es muy conocido el «teorema de la historia de la ciencia" de Sarton, que avala esta perspectiva de la ciencia como conocimiento positivo sistematizado. El teorema dice: "Definición. La ciencia es conocimiento positivo sistematizado, o lo que ha sido considerado así en épocas y lugares diferentes. Teorema. La adquisición y sistematización del conocimiento positivo son las únicas actividades humanas que son verdaderamente acumulativas y progresivas. Corolario. La historia de la ciencia es la única historia que puede ilustrar el progreso de la humanidad». George SARTON, The Study of the History of Science, Cambridge (Mass.): Harvard University Press, 1936, p. 5. Cit. (entre otros) por Arnold ThaCKRAY y Robert K. MERTON, "On Discipline Building: The Paradoxes of George Sarton», Isis, 63 (4) (dic. 1972), pp. 472-495, esp. p. 479.

7 George SARTON, "The History of Science versus the History of Learning", en Paul Henle, Horace M. Kallen y Susanne K. Langer (eds.), Structure, Method and Meaning: Essays in Honor of Henry M. Sheffer, Nueva York: The Liberal Arts Press, 1951, pp. 145-151, esp. p. 146. 
de física en esa educación general es «(...) librarse de la actitud históricamente ingenua de que lo que uno lee en cualquier libro tiende a ser verdad ${ }^{8}$.

Más allá de la órbita de Sarton, y a principios de los años cuarenta, algunas instituciones como la American Association for the Advancement of Science se habian puesto a trabajar en las necesidades de una educación científica apropiada al nivel de licenciatura (i.e., el nivel de la conocida como "educación general»). Uno de los participantes en las deliberaciones de la asociación, L. W. Taylor, profesor de fisica en el Oberlin College de Ohio, reflexionaba sobre el contenido humanístico de una educación apropiada en ciencias y acerca del modo de adquirirlo y transmitirlo. Como solución decía: «(...) de los diversos enfoques de este problema, el que me convence como más prometedor es el de que, aunque se mantenga en lo esencial el plan actual de cursos generales de ciencias y las líneas temáticas básicas, se sitúen esos temas en el escenario de la historia de su desarrollon'. Taylor citaba unas frases del químico y por entonces presidente de Harvard, James B. Conant, quien decía: «Una gran parte del significado del conocimiento acumulado se encuentra al comprender del proceso mediante el cual fue acumulado" ${ }^{10}$.

Conant era un defensor de la historia de la ciencia como instrumento de formación de los futuros líderes de la cultura y la política norteamericanas. No todo el mundo puede ser cientifico, recordaba Conant, pero en una cultura en la que la ciencia ocupa un lugar tan primordial, una formación adecuada en su trasfondo común es sumamente valiosa. Tarde o temprano, dice Conant, el líder debe hacer frente a problemas del movimiento que encabeza; el futuro de la ciencia de toda una nación sería un buen ejemplo. Su solución dependerá en ese caso de una correcta familiarización con la ciencia y su modo de trabajo, de acción y de solución de problemas ${ }^{11}$. Para Conant, la historia de la ciencia era capaz de proporcionar una comprensión de la ciencia mucho más profunda y general que la que aportaba la especialización en una única de las disciplinas científicas. El citado trasfondo común de la ciencia se aprecia con mucha mayor facilidad. Este trasfondo es el

${ }^{8}$ I. Bernard COHEN, "A Sense of History in Science", American Journal of Physics, 18 (6) (sept. 1950), pp. 343-359, esp. 349.

9 Lloyd W. TAYLOR, "Science in General Education at the College Level", Science, 91 (2372) (14 jun. 1940), pp. 560-565, esp. p. 564.

${ }^{10}$ Frase de James B. CONANT en el Bulletin of the Association of American Colleges, 23 (1937), p. 43. Cit. en Taylor, op. cit., p. 564.

11 James B. CONANT, On Understanding Science: An Historical Approach, New Haven (Conn.): Yale University Press, 1947, esp. pp. 3-4. 
modo en que cada científico individual ataca un determinado problema; y existe un sustrato común a todo científico, sea cual sea su disciplina. Ese trasfondo no es sin embargo algo que nos revele con éxito la perspectiva de la filosofla de la ciencia, con su confianza en el orden lógico presente en las teorías tal cual se enseñan en las aulas de ciencias. La historia nos muestra aspectos de esa actitud común que son esenciales, pero que permanecen ocultos bajo la perspectiva filosófica, y que debemos conocer (sobre todo con los objetivos de Conant en mente) ${ }^{12}$.

Conant era especialmente crítico con la canónica The Grammar of Science de Karl Pearson. En principio, Conant sí que compartía dos ideas de Pearson. La primera era la de que en el descubrimiento de las leyes científicas participa la imaginación creativa. La segunda, que los científicos se encuentran al mismo nivel en lo referente a la crítica de sus resultados; la autocrítica es, de hecho, una herramienta de trabajo básica. Sin embargo, había una tercera idea de Pearson que, para Conant, era rotundamente incompleta y sumamente perniciosa para educar al público en los métodos de la ciencia. Tal idea, que ocupaba la mayor parte de la discusión de Pearson por ser fundamental en su perspectiva del método, consistía en que el método científico posee un rasgo inequívoco principal: el primer paso del científico es clasificar hechos y extraer correlaciones de ellos sin la participación de sus creencias y emociones. Para Conant, esta idea tiene poco que ver con lo que hace un cientifico ${ }^{13}$. Como principio subyacente a esta idea, Conant veía una tendencia a aislar los pasos a seguir (i.e., el método explícito) del científico, cuando la única constante visible en la historia de la ciencia era la disposición del científico a desarrollar un plan de acción de cara a resolver un problema también de su elección. No sólo se trata de que la única constante visible sea psicológica en lugar de lógica, sino de que la ciencia se caracteriza en general por la disposición variable del científico, no por un cúmulo de rasgos todos ellos únicos y uniformes en la historia.

Precisamente, Conant empleaba casos históricos porque en ellos se veían los modos en que un científico del pasado había enfocado el tratamiento de un problema. Tales modos se dividían (de forma voluntariamente gruesa) en "tácticas" y «estrategias». El buen táctico sabía trabajar en el seno de una teoría consolidada y

12 Ibid., pp. 10-12.

${ }^{13}$ Ibid., p. 14. Conant (ibid., pp. 111 y 114) cita The Grammar of Science DE PEARSON por su edición "Everyman" (orig. $2^{2}$ ed., Londres: Adam and Charles Black, 1900), esp. p. 37. 
se manejaba bien a la hora de trabajar en ella teórica y experimentalmente y de desarrollar el instrumental apropiado. El estratega, en cambio, podia fijarse un objetivo más allá de dicha teoría y, en pro del mismo, dar pasos poco coherentes desde el seno de la ortodoxia vigente. Ambos extremos, que sólo en casos muy raros habían caracterizado la obra de un científico en particular, constituían los prototipos de un par de actitudes que todo científico incluía en mayor o menor medida a la hora de trabajar en un problema. La observación y la experimentación dependen de esos modos de trabajo; vienen precedidas por ellos. La comprensión de la ciencia no consiste, por lo tanto, en conocer qué relación existe entre la experiencia y la teoría, sino en analizar de qué premisas de naturaleza psicológica, creativa, surge el plan específico de cada científico a la hora de establecer esa relación entre teoría y experiencia ${ }^{14}$. Conant disponía finalmente de un esquema cíclico en el que los esquemas conceptuales se sucedían unos a otros a causa de un mejor conocimiento de los fenómenos debido a una técnica instrumental más desarrollada. En el trasfondo de ese ciclo descansa una imagen de la mente creativa del científico que no se sometía con facilidad al cliché filosófico (metodológico) por entonces vigente.

En lo que respecta a la invitación a examinar la historia de la ciencia para corregir la perspectiva ingenua del desarrollo de la ciencia, Kuhn podría contemplarse como un "seguidor" de Sarton, Cohen y Conant, entre muchos otros. (No en vano, Kuhn fue ayudante de Conant en Harvard y se benefició de los conocimientos historiográficos de I. B. Cohen en su etapa formativa como historiador.) Sin embargo, si hemos introducido la palabra «seguidor» entre comillas es porque la perspectiva de Kuhn sólo comparte algunos rasgos reconocibles con la de Conant que se puedan traducir en influencias, mientras que prácticamente no existen vínculos con la de Cohen y mucho menos con la de Sarton.

${ }^{14}$ Sobre todo ello, cf. CONANT, On Understanding Science, loc. cit., esp. pp. 8-9 y 18-19. El capitulo III de esta obra (ibid., pp. 65-97) es especialmente revelador del punto de vista de Conant aquí descrito. En ese capítulo, Priestley y Lavoisier representan de forma explícita (por primera y única vez) al táctico y al estratega, respectivamente, y muestran cómo la diferencia entre ambas figuras radica en sus respectivos planteamientos del plan de acción teórico y, sobre todo, experimental. Existe una imagen paralela (seguramente germinal en el pensamiento de Conant) a la de Priestley y Lavoisier en dos de los mentores de química de ConanT en Harvard: el Premio Nobel Theodore W. Richards y Elmer P. Kohler. Sobre ello, cf. James B. ConANT, My Several Lives: Memoirs of a Social Inventor, Nueva York: Harper \& Row, 1970, p. 34; en general cf. ibid., cap. 4 (pp. 27-40), esp. pp. 27-37, para una descripción del trabajo de ambos químicos, que puede compararse con la diferencia táctico/estratega arriba mencionada. 
Bajo la perspectiva de Kuhn, la tarea de la historia de la ciencia no consiste en registrar los grandes hallazgos con nombres y fechas al modo de Sarton; la historia de la ciencia no es un mero monumento a la grandeza humana, sino una tarea de investigación. Para Kuhn, de hecho, aunque fuera un monumento, ni siquiera tendría por qué ser el principal, como lo es para Sarton ${ }^{15}$. Algo parecido lo aleja de Cohen, aunque éste no sea un historiador positivista a la manera de Sarton. Como historiador, Kuhn no se fija la tarea de completar los grandes temas de la historia de la ciencia. A diferencia de Cohen, Kuhn no es especialista en ningún gran científico, aunque conoce bien la obra de Boyle, Newton, Carnot y Planck. Kuhn se caracteriza más bien por historiar el recorrido y el ciclo vital de las herramientas de análisis de la naturaleza que en otras épocas constituyeron la punta de lanza de la investigación científica ${ }^{16}$. Para Kuhn los protagonistas son los conceptos y los recursos científicos disponibles en un episodio histórico determinado. $\mathrm{E}$ incluso el principio y el fin del propio episodio viene dado por la vigencia de esos recursos y conceptos. La principal diferencia con la labor de Cohen que este enfoque conlleva es que hay un objetivo argumentativo (con frecuencia extra-historiográfico) que guía la investigación, que es además la fuente de los recursos de selección y de análisis de los episodios históricos. Así, la opinión de Kuhn sobre la obra de Cohen, más allá de la carga peyorativa que lleva impresa, no debería extranaarnos demasiado: "(...) la selección de información de Bernard [Cohen] parece a menudo insuficientemente controlada, y el uso que hace de la información que ha acumulado suele ser bastante insustancial y superficial. Muchas de las tareas en las que se pone a trabajar requieren una mente inmensamente más analítica y crítica que la suya. El resultado de sus esfuerzos es demasiado a menudo un compendio útil que claramente trata de ser mucho más» ${ }^{17}$.

15 A este respecto cf. las palabras de Kuhn sobre Sarton en Aristides BaLTAS, Kostas Gavroglu, Vassiliki Kindi y Thomas S. KUHN, "A Discussion with Thomas S. Kuhn", Neusis: Journal for the History and Philosophy of Science and Technology, 6 (1997), pp. 143-198. Citamos por la traducción de José Romo de la reedición de la entrevista en Thomas S. KUHN, El camino desde la estructura: Ensayos filosoficos, 1970-1993, con una entrevista autobiográfica, James Conant y John Haugeland (eds.), trad. Antonio Beltrán y José Romo, Barcelona: Paidós, 2002 (orig. 2000), tercera parte (pp. 301-373), pp. 328-329.

16 Ibid., p. 369. En esa página, Kuhn menciona que "(...) sólo uno de mis estudiantes (...), Jed Buchwald, hace el tipo de historia de las ideas analíticas que yo hago y me gusta hacer».

${ }_{17}$ Carta de Thomas Kuhn a Samuel M. Grupper, 6 de septiembre de 1990, Thomas S. Kuhn Papers, Institute Archives and Special Collections, MIT, Cambridge (Mass.), MC 240, caja 21, carpeta 18. Grupper era el ayudante de dirección del programa de becas MacArthur, a una de las cuales Cohen era candidato. Está claro que Kuhn no colaboró demasiado a que la beca le fuera concedida. La enemistad entre Cohen y Kuhn era ya ańeja en los años noventa. La corresponden- 
En cambio, este interés por el estudio de las herramientas pasadas del análisis científico (desde un prisma en sí mismo no menos analítico) acercaba a Kuhn a Conant, sin que, eso sí, podamos decir en justicia que aquél «siga» a este último. Conant fue una de las dos únicas personas cuya inteligencia y personalidad Kuhn siempre estimó en grado sumo. (La otra fue su padre, Samuel L. Kuhn.) ${ }^{18}$ Conant le sirvió a Kuhn de mentor y de promotor de su propia carrera profesional en la historia de la ciencia. Lo introdujo en la enseñanza general de Harvard (i.e., la dedicada a los alumnos de primer año, una enseñanza muy laxa y humanística) y lo propuso como fellow de la Society of Fellows de Harvard, una de las instituciones de mayor prestigio del país en aquella época, donde se formaron algunas de las principales personalidades de las letras y las ciencias de la segunda mitad del siglo XX, como B. F. Skinner, John Bardeen, W. V. Quine y Noam Chomsky ${ }^{19}$. Al igual que Conant, Kuhn no provenía de la historia de la ciencia, y ésta era un medio para lograr otros fines —en su caso filosóficos, en el de Conant, educativos- y no un fin en sí misma. Ambos compartían la tendencia a una reflexión acerca de las consecuencias de conocer e investigar la historia de la ciencia. Quizá por ello, Conant y Kuhn mantuvieron durante muchos años una relación intelectual muy fluida (no exenta de debates y discrepancias, naturalmente). Sin embargo, las concepciones que cada uno de ellos poseía de la naturaleza de la ciencia divergían desde la

cia entre Crane Brinton (profesor de historia en Harvard y presidente de su Society of Fellows por entonces) y Kuhn durante el verano y los comienzos del otono de 1963 pone de manifiesto que existía una clara animadversión entre Cohen y Kuhn, pese a que años antes Cohen había ayudado a Kuhn a formarse como historiador e incluso le había ofrecido la introducción a los escritos inéditos de óptica de Newton, además de ser probablemente quien sugirió el nombre de Kuhn para escribir la monografía de historia de la ciencia (Structure) para la International Encyclopedia of Unified Science de Carnap, Morris y Frank. (Sobre esta última recomendación de Cohen, cf. Baltas et al., op. cit., p. 339.) Sin embargo, Brinton deja claro que había celos intelectuales por parte de Cohen, lo cual era un impedimento a la reentrada de Kuhn en Harvard después de su salida en 1956. Como Brinton le decía a Kuhn, «(...) creo - y esto es completamente confidencial-que la opinión que Bernard [Cohen] tiene de ti todavía es un verdadero obstáculon.Y añadía que Cohen tenía el tipo de “(...) sentimiento de inferioridad que la mente narrativa, erudita y acumulativa tiene por tu mente analítica». Carta de Crane Brinton a Thomas S. Kuhn, 12 de junio de 1963, Thomas S. Kuhn Papers, loc. cit., caja 14, carpeta 15. Para otros indicios de esta mala relación, cf. el resto de la correspondencia Brinton-Kuhn de esta misma carpeta.

${ }^{18}$ Cf. Baltas et al., op. cit., p. 305.

${ }^{19}$ Ibid., pp. 322-323. Sobre la Society of Fellows de Harvard puede consultarse el artículo (sin firma de autor) "The Society of Fellows: The Story of Fifteen Years", Harvard Alumni Bulletin, 50 (24 abr. 1948), pp. 593-599, asl como el texto de George C. Homans y Orville T. BAILEY, The Society of Fellows, Harvard University, 1933-1947, Cambridge (Mass.): Harvard University Press, 1948. 
raíz. Esa diferencia nos impide hablar de Kuhn como un «seguidor» de Conant. Pero la diferencia es muy sutil, interesante, y no menos importante.

En la raíz de la que hablamos, el origen de la discrepancia entre Conant y Kuhn, hemos de situar un nombre clave, el de Percy W. Bridgman ${ }^{20}$. Bridgman fue un físico experimental al que le fue concedido el Premio Nobel de física por sus experimentos con altas presiones. Bridgman fue además un educador sobre termodinámica y relatividad de las jóvenes generaciones de f́́sicos que sucedieron a la consolidación de la mecánica cuántica (y, en general, de la física teórica) en los Estados Unidos - una de las cuales fue la del propio Kuhn-. Por último, lo que es más relevante para nosotros en este momento, Bridgman escribió uno de los clásicos del análisis metodológico de la época, The Logic of Modern Physics (1927). Desde luego, el libro de Bridgman no transformó el trabajo de los físicos, aunque sí les resultó convincente y representativo del proceso real de consolidación teórica y conceptual. Tuvo asimismo su influencia entre los filósofos, pero también fue muy criticado por éstos debido a la formulación, para muchos insatisfactoria, de la herramienta metodológica fundamental que introducía y en la que se basaba: el análisis operacional.

El análisis operacional —según el propio Bridgman, incorrectamente denominado "operacionalismo" e incluso "operacionismo" ${ }^{21}$ - era un criterio para averiguar el contenido empírico de los conceptos físicos y ver si éstos podían considerarse conceptos "con significado". El criterio consistía en averiguar si existían operaciones independientes por medio de las cuales tales conceptos se correspondían con valores numéricos productos de la medición. Así, si el concepto de "longitud" posee significado es porque contamos con mecanismos de

${ }^{20}$ Para los detalles biográficos mencionados a continuación, cf. Edwin C. KEMBLE, Francis BirCH y Gerald HolTON, "Percy Williams Bridgman», en Charles C. Gillispie (dir.), Dictionary of Scientific Biography, vol. 1, Nueva York: Charles Scribner's Sons, 1981 (orig. 1970), pp. 457461; Albert E. MOYER, «P. W. Bridgman's Operational Perspective on Physics, Part I: Origins and Developmentw, Studies in History and Philosophy of Science, 22 (2) (1991), pp. 237-258; Albert E. MOYER, "P. W. Bridgman's Operational Perspective on Physics, Part II: Refinements, Publication, and Reception", Studies in History and Philosophy of Science, 22 (3) (1991), pp. 373397; Maila L. WALTER, Science and Cultural Crisis: An Intellectual Biography of Percy Williams Bridgman (1881-1961), Stanford (Cal.): Stanford University Press, 1990.

${ }^{21}$ Según Bridgman, esos vocablos parecían indicar que el análisis operacional era una teoría del conocimiento o del significado, cuando en realidad su intención era proporcionar un método de aclaración de conceptos. Sobre este rechazo, cf. Percy W. BRIDGMAN, "Operational Analysis", Philosophy of Science, 5 (2) (1938), pp. 114-131, esp. pp. 114-117. 
medida estándar (por ejemplo, una vara de medir) que a su vez está basada en algún sistema, también estándar, de medida. Este criterio posee una importante consecuencia, y es que, aunque utilicemos el mismo término para hablar de conceptos cuyo significado proviene de grupos de operaciones diferentes, en realidad dicho término expresa en cada caso conceptos también diferentes. Así, en astronomía y en cartografía se emplean métodos de medida óptica para establecer longitudes. En este caso, aunque empleemos de forma sinónima "longitud" para el resultado de esta medición y para el producto del empleo de varas de medir, y psicológicamente consideremos que estamos ante el mismo concepto, la teoría física, nos dice Bridgman, debería distinguir convenientemente entre uno y otro concepto. Cada ámbito de los fenómenos clama por una serie de operaciones particulares, y toda supuesta sinonimia hay que probarla. Esta distribución rige el uso de los conceptos en el seno de una teoría física ${ }^{22}$.

En la perspectiva de Bridgman, los conceptos (i.e., la teoría) no son una imposición a los fenómenos. La presencia de constantes físicas (obviamente irreducibles) en nuestras leyes indica que hay un orden fenoménico que se resiste a toda imposición teórica. La física debe tratar de comprender ese orden y dominarlo, pero sin imponerle esquemas a priori. Como podemos ver, el criterio de significado que surge del análisis operacional es literalmente un criterio empirista, razón por la cual dicho análisis fue muy bien recibido por el positivismo lógico, entre otras corrientes filosóficas. La relación entre los resultados de medición rige la manipulación de conceptos. Bridgman estaba sumamente interesado en establecer estas pautas, sobre todo en una época de cambio como la atravesada por la física en el primer tercio del siglo XX. Para Bridgman, no había problema en el uso común de los conceptos de "tiempo», "espacio» o "causa» dentro de la mecánica clásica, pero el uso indiscriminado de estos conceptos podía acarrear problemas en el seno de las nuevas teorías, como la de la relatividad o la mecánica cuántica ${ }^{23}$. $\mathrm{El}$ análisis operacional era principalmente una vía de cautela y de terapia que pretendía evitar que la teoría física no se desarrollara de forma natural ${ }^{24}$.

22 Percy W. BRIDgman, The Logic of Modern Physics, Nueva York: Macmillan, 1928 (orig. 1927), cap. 1, esp. pp. 9-25.

${ }^{23}$ Cf., p. ej., ibid., pp. 186 (nota), 191-192 y 202-203.

${ }^{24}$ Bridgman fue también, y debido a la misma perspectiva, uno de los principales teóricos del llamado "análisis dimensional», mediante el cual se establece la relación de proporción cuantitativa que existe entre las diversas cantidades físicas, ya sean básicas (como la longitud) o secundarias, también llamadas "dependientes" (como la velocidad). Una vez conocidas las dimensiones de una determinada cantidad podemos usarlas, por ejemplo, como guía de investigación. Sobre ello, cf. 
La historia de la ciencia adopta una forma característica desde la perspectiva del análisis operacional. Bajo este prisma, hay dos fuentes principales del desarrollo científico. Una de ellas es el ingenio del científico individual. La otra, el dominio de todas las variables de experimentación y medida, el desarrollo del llamado "experimento controlado". Y si el ingenio, la creatividad, son propios de todo científico desde el mundo antiguo, el experimento controlado es una creación de la filosofía experimental del siglo XVII. Como dirá Bridgman, la historia de la ciencia presenta dos épocas visibles:

Se pueden distinguir dos épocas científicas. La primera va desde Galileo y Newton hasta la primera parte del siglo actual [XX]. (...) La segunda época comienza con este siglo, con las revoluciones en el pensamiento científico que implicaron la teoría de la relatividad y la mecánica cuántica. (...).

La primera época científica comenzó con lo que era en esencia un nuevo truco de técnica intelectual: el experimento controlado. Este está ahora tan aceptado como algo natural que la recuperación del punto de vista de los días antiguos requiere un gran esfuerzo de la imaginación. En aquellos días la naturaleza era desesperadamente compleja, porque todavía ni siquiera se había previsto que existieran las leyes de la mecánica y de la química. Por lo tanto, no era en absoluto obvio que fuera posible crear situaciones sencillas de manera artificial que fueran significativas porque se repitieran en situaciones más complejas. En realidad, no estaba claro qué constituía la simplicidad de una situación. (...) El resultado final de muchas décadas de ensayo y error, de meditación y enseñanza, fue el desarrollo de una nueva herramienta para el uso de la inteligencia, que por sí misma ha hecho posible la ciencia y la tecnología actuales ${ }^{25}$.

Según esta perspectiva, la historia de la ciencia se inicia con los pioneros de la preparación de experimentos controlados. Robert Boyle, asumido padre de la filosofía experimental, es, junto al ideólogo por excelencia, Francis Bacon, la bisagra sobre la que rota la indagación filosófica de la naturaleza y

Percy W. Bridgman, Dimensional Analysis, New Haven (Conn.): Yale University Press, 1931 (orig. 1922), cap. 2, esp. pp. 17-23.

${ }^{25}$ Percy W. Bridgman, "The Prospect for Intelligencen, The Yale Review, 54 (1945), pp. 444-461, esp. p. 451. 
el punto de partida de la primera forma de investigación propiamente científica (i.e., tal y como hoy la conocemos) ${ }^{26}$.

Si reexaminamos los casos históricos de Conant en On Understanding Science observaremos que el primero de ellos se titula "Illustrations from the 17th Century "Touching the Spring of the Air"”. En él Conant habla de cómo las nuevas técnicas de experimentación provocaron el desarrollo de nuevas nociones relativas a la naturaleza del aire (como el «mar de aire» y el «resorte del aire») ${ }^{27}$. La nueva experimentación, las nuevas técnicas, proporcionaron el material observacional para los nuevos conceptos. Parafraseando a Conant, en este ejemplo (cuya figura primordial es Robert Boyle) se captan a la perfección las dificultades de la experimentación y la naturaleza e importancia del experimento controlado ${ }^{28}$. Boyle permite que captemos cómo la producción de conceptos depende del manejo de los aspectos relevantes de una situación real simplificada (el experimento bajo control) y cómo los nuevos conceptos dependen a veces de la captación de una diferencia cuantitativa mínima (lo cual precisa de ingenio, sensibilidad hacia la situación experimental y buen diseño del control experimental) ${ }^{29}$. Para Conant, Robert Boyle se adelantó varios siglos a su propia época cuando distinguió su concepto de aire como fluido elástico de la explicación en sí de esa elasticidad mediante imágenes y modelos. A este respecto, dice Conant:

Casi todos sus contemporáneos estaban interesados en gran medida en teorías sobre el universo y se preocupaban poco o nada por si éstas podían someterse a examen experimental. Insistir en la necesidad de distinguir una afirmación sometible a prueba experimental de una propuesta de representación de los hechos observables mediante ciertas palabras o diagramas se ha convertido en parte de la filosofia de la ciencia aceptada sólo en este siglo. Éste bien podría ser el punto apropiado en el curso para hacer referencia al

${ }^{26}$ Para una revisión de la función y naturaleza real del diseño y el informe experimental en los trabajos de Robert Boyle, cf. el articulo de Carlos Solfs, "Erudición, magia y espectáculo: El juicio de la República de las Letras sobre Athanasius Kircher», en esta misma monografía. Cf. también los textos ya clásicos de Steven SHAPIN y Simon SCHAFfer, Leviathan and the Air Pump: Hobbes, Boyle and the Experimental Life, Princeton: Princeton University Press, 1985, y de Steven SHAPIN, A Social History of Truth: Civility and Science in Seventeenth-Century England, Chicago: The University of Chicago Press, 1994.

${ }^{27}$ CONANT, On Understanding Science, loc. cit., cap. II, pp. 29-64.

${ }^{28}$ Ibid., p. 59.

${ }^{29}$ Cf. ibid., esp. pp. 44-50 y 56-59. 
punto de vista operacional expuesto con claridad por el profesor Bridgman en su Logic of Modern Physics. ${ }^{30}$

On Understanding Science es, por lo tanto, una "historia operacional" con tonos pedagógicos; el proyecto de Conant para enseńar ciencia mediante su historia. Conant admitió a Kuhn en 1961 lo siguiente: "Intenté presentar una definición "operacional" de progreso (en términos de conocimiento acumulativo) en "Understanding Science". (...) Todavía creo que la idea básica es válida y la estoy usando de nuevo en mis escritos sobre educación" ${ }^{31}$. La ciencia a enseñar era la presente en The Logic of Modern Physics y correspondía al análisis operacional. La discrepancia con Kuhn comienza en este punto; en especial, en lo referente al papel de los conceptos en el desarrollo de la teoría física y a la naturaleza de la clasificación.

\section{La vida secreta de los conceptos científicos}

Thomas S. Kuhn se educó como físico en un entorno en el que la obra de Bridgman era representativa de la vaga concepción que cada físico teórico poseía de su propio ejercicio de investigación. Kuhn pertenece a un estrato generacional para el que la mecánica cuántica era el vocabulario primordial de la física teórica y para el que la traducción (si podemos llamarla así) de los trabajos en términos cuánticos a un lenguaje clásico (i.e., a la física clásica) era un recurso un tanto forzado y en ocasiones carente de sentido. Para la generación inmediatamente anterior a la de Kuhn, la de, por ejemplo, John H. Van Vleck y Edwin C. Kemble (el primero, director de tesis de Kuhn), ese orden de prioridad estaba invertido por lo que ambos (como muchos otros) solían buscar las equivalencias clásicas de los resultados cuánticos ${ }^{32}$.

${ }^{30}$ Ibid., p. 48.

31 Carta de James B. Conant a Thomas S. Kuhn, 11 de julio de 1961, Thomas S. Kuhn Papers, loc. cit., caja 25, carpeta 53, pp. 3-4.

${ }^{32}$ Agradezco a Philip W. Anderson, físico de la generación de Kuhn (y Premio Nobel de física), sus comentarios a este respecto. (Philip W. Anderson, comunicación personal vía e-mail, 12 de noviembre de 2002.) Para una autoconfesión de las dificultades de Kemble con el formalismo abstracto de la mecánica cuántica (en el que no obstante trabajó con éxito), cf. Edwin C. Kemble, entrevista con Thomas S. Kuhn, 1-2 de octubre de 1963, Archives for History of Quantum Physics, Oral History Records, p. 16. Cf. también Gerald HolTON, "On the Hesistant Rise of Quantum Physics Research in the United States", en sus Thematic Origins of Scientific Thought: Kepler to Einstein, Cambridge (Mass.): Harvard University Press, 1988, pp. 147-187, esp. p. 169. 
A pesar de que, como decimos, había una noción bastante vaga del trasfondo metodológico de la física, no faltaban las reflexiones esporádicas al respecto. Para el citado Kemble, por ejemplo, la teoría física avanza por un terreno de abstracción progresiva ajena a las paradojas que pueda forzar en el sentido común. Para Kemble es cierto, por un lado, que la teoría física parte de los objetos cotidianos para reconstruir el mundo de los fenómenos físicos. Pero, por otro lado, en determinado momento a esos objetos les suele faltar la flexibilidad necesaria para afrontar las nuevas particularidades conocidas de la materia, el tiempo y el espacio. Surgen así perspectivas que, como la dualidad onda-partícula o el principio de incertidumbre, apoyan una descripción exitosa de los fenómenos, aunque también reducen la claridad de las explicaciones y, sobre todo, lo que podríamos llamar su "visualizabilidad". Sin embargo, dice Kemble, ocuparse del sentido común y de sus problemas con la física no es la tarea del físico sino del filósofo ${ }^{33}$. El físico no busca "más sentido común", sino aumentar el conocimiento detallado y empíricamente sólido del mundo de los fenómenos. Y para Kemble ambos mundos, el fenoménico y el, llamémoslo así, socio-psicológico (o de usentido común" ), son en principio separables ${ }^{34}$.

Esta diferencia entre mente y mundo es representativa de los físicos de la generación de Kuhn. La podríamos llamar "positivista", pero tendríamos que matizar algo al respecto antes de hacerlo. Al igual que Conant observaba en Robert Boyle, para estos físicos los mecanismos de explicación se pueden aislar de los de descripción y predicción, y no es necesario prescindir de ninguno de ellos ni, como pretendieron los positivistas lógicos, el de explicación debía subsumirse o fundirse con el de descripción, haciendo desaparecer con ello los supuestos ontológicos auxiliares. Estos últimos supuestos son tremendamente útiles para la investiga-

${ }^{33}$ Aunque muy reciente, un texto de Steven Weinberg ilustra bien esta actitud: «Esto me parece un poco como decir que, puesto que los granjeros no pueden definir a las vacas o la diferencia entre las vacas y, por ejemplo, los búfalos, uno debe dudar de la existencia objetiva de las vacas. Creo que no es el trabajo de los granjeros definir a las vacas; ése es el trabajo de los zoólogos. Del mismo modo, no es el trabajo de los físicos u otros científicos definir la verdad; ése es el trabajo de los filósofos. Si no han hecho ese trabajo, tanto peor para ellos. Pero al igual que el granjero generalmente reconoce las vacas cuando las ve, los científicos normalmente reconocemos la verdad cuando la vemos". Steven WeInberg, Plantar cara: La ciencia y sus adversarios culturales, trad. J. V. Mayoral, Barcelona: Paidós, 2003 (orig. 2001), p. 111. El párrafo está provisto de toda la acidez propia de su autor y además está dirigido precisamente contra la filosofía de Kuhn.

${ }^{34}$ Cf. Edwin C. KeMBLE, "Operational Reasoning, Reality, and Quantum Mechanics", Journal of the Frankin Institute, 225 (3) (1938), pp. 263-275, esp. pp. 265-266. 
ción científica, pero debe quedar claro que están al final de la lista de lo que un fisico debe respetar a la hora de desarrollar una teoría. Los citados supuestos ayudan, sirven a la investigación de los fenómenos, no se imponen a éstos.

Páginas atrás vimos esta misma idea sobre el papel de los conceptos en Bridgman. Para Bridgman todo desarrollo teórico ha de cumplir con unos preceptos de significado bien representados en el análisis operacional. No es desdeñable el difícil trabajo de encaje teórico y conceptual de los nuevos hallazgos. De hecho, los conceptos se reordenan sólo con enorme dificultad, ya que normalmente se suele tejer una red teórica coherente que además suele frenar su propia modificación (debido en parte a esa misma coherencia) ${ }^{35}$. Pero tal modificación es prioritaria siempre que provenga de la investigación puramente empírica. Lo único necesario son nuevos conceptos auxiliares ${ }^{36}$. Los conceptos, como acabamos de indicar más allá de Bridgman, son también para él una ayuda, nunca un obstáculo.

Kuhn se educó en un ambiente en el que el operacionalismo era algo "natural". Para los físicos de la época lo que decía Bridgman tenía completo sentido. «Las ideas positivistas," recordaba Kuhn en 1965, uincluyendo la perspectiva especificamente operacional de Bridgman, se palpaban en la atmósfera de Harvard en los años en que yo estaba estudiando la licenciatura, (...) [con lo que] no puedo haber encontrado nada en el libro [de Bridgman, The Logic of Modern Physics] que fuera realmente nuevo para mí en espíritu» . Kuhn incluso advierte que "(...) estoy segu-

${ }^{35}$ Como sefiala Bridgman, "Hallar conceptos que se ajusten a la naturaleza se parece mucho a resolver un crucigrama. En el crucigrama puede haber algunas partes del esquema que rellenamos completa y fácilmente, pero a veces encontramos partes en las que podemos llenar todo excepto una o dos definiciones obstinadas, con lo que estamos seguros de ir tras la pista correcta y nos devanamos los sesos en la búsqueda de la palabra perdida, cuando con una ráfaga de inspiración vemos que las palabras obstinadas se pueden ajustar mediante un cambio completo en aquéllas que ya hemos aceptadon. BRIDGMAN, The Logic of Modern Physics, loc. cit., pp. 202-203.

${ }^{36}$ Para Conant, una teoría sólo cae si al peso de los hechos contradictorios a ella se le suma la presencia de una nueva teoría, concepto o grupo de ellos: "Podemos exponer como uno de los principios aprendidos de la historia de la ciencia que una teoría sólo es derrocada por una teoría mejor, nunca meramente por hechos contradictorios. Primero se llevan a cabo intentos de reconciliar los hechos contradictorios con el esquema conceptual existente mediante alguna modificación del concepto. Sólo la combinación de un nuevo concepto con hechos contradictorios con las antiguas ideas da finalmente lugar a una revolución científican. CONANT, On Understanding Science, loc. cit., pp. 36-37. Al conocedor de la obra de Kuhn estas palabras le parecerán sacadas del propio Structure. Espero que lo relatado en este artículo consiga revelar las diferencias entre los dos. 
ro de que, como muchos de mis contemporáneos, lo consideré una liberación al principio (...) $)^{37}$. Sin embargo, Kuhn nunca fue un seguidor de Bridgman como sí lo fue, por ejemplo, Conant. Kuhn seguía el tono de interés sólo laxo de los físicos coetáneos por el texto de Bridgman. Para ellos, este libro era principalmente un interesante y convincente divertimento como toda filosofia de la ciencia, y ya entonces hubieran asentido al aforismo de Weinberg de que "la filosofia de la ciencia viene a ser tan útil para los científicos como la ornitología para los pájaros»” ${ }^{38}$.

Kuhn sin embargo no llegaba tan lejos. Su laxitud de interés se refería en exclusiva al texto de Bridgman, pero no al resto de la filosofía de la ciencia. A decir verdad, mucho antes de establecer contacto con Conant y de llegar a conocer y mucho menos apreciar el valor de- la historia de la ciencia, Kuhn coqueteó con la filosofía de la ciencia hasta el extremo de tratar de abandonar su física teórica por ese nuevo campo.

Ya durante la licenciatura, Kuhn comenzó a interesarse por la filosofía en general. En el primer año de estudios (1940/41) se matriculó en una historia de la filosofía con Raphael Demos, probablemente estimulado por su tío político, Adolph Oko, un estudioso de la filosofia de B. Spinoza con quien Kuhn habla charlado en más de una ocasión ${ }^{39}$. En esa asignatura, Kuhn trabó un primer contacto con las filosofias de algunos clásicos de Aristóteles a Kant ${ }^{40}$. La idea de precondiciones (i.e., categorías a priori) del conocimiento de este último le llamó tanto la atención que en un trabajo de redacción para una asignatura de inglés del curso siguiente (1941/42) esbozó un programa alternativo al kantiano para la estimación de la capacidad de conocimiento con fundamento empirista en el que los conceptos eran puramente auxiliares y los datos comandaban la investigación de la naturaleza ${ }^{41}$. El plan era acorde a los presupuestos filosóficos de los físicos que aca-

${ }^{37}$ Carta de Thomas S. Kuhn a Edward T. Robinson, 8 de marzo de 1965, Thomas S. Kuhn Papers, loc. cit., caja 4, carpeta 4, pp. 1-2. E. T. Robinson era un estudiante de historia de la ciencia en Harvard que por entonces estaba preparando una "Senior Honors Thesis" sobre la influencia de Bridgman y sus ideas operacionales en el desarrollo de la filosofía de la ciencia. Este fue el motivo por el que se dirigió a Kuhn.

38 Cit. en LABINGER y Collins (eds.), op. cit., p. 4. Por lo visto el aforismo podría haberlo dicho Richard Feynman por primera vez (ibid., nota 1).

${ }^{39}$ Roger Kuhn (hermano menor de Thomas S. Kuhn), comunicación personal vía e-mail, 12 de octubre de 2001; Baltas et al., op. cit., p. 263.

40 Ibid., p. 264.

${ }^{11}$ Kuhn reflejó ese programa en un trabajo titulado "The Metaphysical Possibilities of Physics», ca. 1942, Thomas S. Kuhn Papers, loc. cit., caja 1, carpeta 3, esp. pp. 3-4 y 9. 
bamos de ver. Sin embargo, Kuhn debía continuar profundizando a partir de él en el modo en que los datos señalan el camino de la inducción. ¿Cómo es posible, se preguntaba Kuhn entonces, que los datos requieran determinados conceptos? Y así, ¿qué grado de necesidad acarrearían tales conceptos? Es decir, si son suplantables, ¿cuándo estaremos seguros de que hemos alcanzado una imagen del mundo? Y de esa manera, ¿cuáles son las capacidades metafísicas de la física? ${ }^{32}$ Estas preguntas rondaban la mente de Kuhn en su segundo año de estudios en Harvard.

No se puede decir que Kuhn fuera un físico anómalo en lo que respecta a este interés. El que más y el que menos había combinado en Harvard, desde tiempo inmemorial, sus intereses científicos con una búsqueda del prestigio intelectual que sólo aportaban las letras. Conant fue un buen ejemplo de ello ${ }^{43}$. Saber resolver una ecuación diferencial era combinable con poder hablar con soltura y seguridad de Dante, Shakespeare, John Donne, Robert Browning, o de la política exterior norteamericana en Europa; más bien era deseable que uno fuera hábil en ese terreno; más todavía que lo hiciera en algunas de las selectas sociedades extra-académicas de la universidad; y ya constituia un buen colofón el que, como en el caso de Conant y más tarde de Kuhn, uno se convirtiera en editor de uno de los periódicos estudiantiles como el Crimson o el Lampoon ${ }^{44}$. Kuhn, que había crecido en un entorno

42 Ibid., pp. 8-11. Hemos extrapolado las preguntas principales que surgen de la propuesta de Kuhn. Kuhn posee un programa de respuesta a esas preguntas, y es la de que si supiéramos mejor cuál es la naturaleza de los datos y, en especial, cuál es la cantidad de información alojada en ellos, podríamos estimar qué conceptos serían necesarios para ocuparse de organizar esa información. Quizá lo más significativo de la investigación radique en la duda implicada en las preguntas con las que hemos tratado de resumir la idea principal del texto de Kuhn: ¿dónde radica el criterio de necesidad —llamémoslo así- de un concepto científico?; ¿qué límites (no sólo cuantitativos) posee la citada «información" empírica? Kuhn se ocupará en años venideros de descubrir las respuestas a estas dos cuestiones. Nos permitimos citar nuestra propia investigación, cuyo apartado $3 . I$ del cap. 2 es uno de los primeros lugares donde se describe con detalle este trabajo de Kuhn de 1942: J. V. MAYORAL, Los pilares de la estructura: Las fuentes del pensamiento de Thomas S. Kuhn y su contexto norteamericano, Tesis Doctoral, Madrid, UNED, 2004, esp. pp. 153-160.

${ }^{43} \mathrm{Al}$ igual que Kuhn, Conant fue editor de uno de los periódicos universitarios, The Crimson, y (también como Kuhn más tarde) presidente de una sociedad literaria de Harvard gran prestigio, The Signet Society. Durante sus estudios de licenciatura aprendió, además de química, en la que brillaba sobradamente, economía, francés, arte y cultura del Renacimiento en Italia, y filosofía, asistiendo en este último caso a las clases de George Herbert Palmer y de George Santayana. Cf. James G. HershBerg, James B. Conant: Harvard to Hiroshima and the Making of Nuclear Age, Stanford (Cal.): Stanford University Press, 1993, cap. 2, esp. pp. 30-33; Conant, My Several Lives, loc. cit., pp. 24-25.

${ }^{44}$ En The Crimson Confidential Guide to Harvard Courses (la guía no-oficial para el estudiante de primer año) de 1940 (Cambridge [Mass.]: The Harvard Crimson, 1940, p. 6), se 
neoyorquino que privilegiaba el prestigio intelectual, se encontraba cómodo en ese terreno en Harvard ${ }^{45}$. Sus estudios de física estaban sazonados con el prestigio del joven cultivado, y la filosofía era sólo una materia más que añadir a esa tendencia a reflexionar más allá de la rutina del aprendizaje físico y matemático.

Lo que ya no era tan común en un físico era que los devaneos con las letras constituyesen un interés serio. El caso de Bridgman era sin duda una excepción. Sin embargo, la filosofía caló en Kuhn lo suficiente como para pensar en dedicarle todos sus esfuerzos. Al final de sus estudios de licenciatura (que había acabado summa cum Laude en física), Kuhn le confesaba a su tía Emma K. Fisher que: «Me inclino (...) cada vez más hacia la universidad, y (...) hacia la filosofía de la ciencia y por medio de ella, más que a la pura física. Pero todavía me encuentro lejos de saber donde acabará [esto] (...) " ${ }^{46}$. Kuhn escribía estas frases poco antes de incorporarse a las investigaciones como físico para el Radar Research Laboratory, sito en Harvard durante la guerra. Durante el periodo bélico, que lo conduciría a Inglaterra y a Francia, Kuhn continuó interesándose por la filosofía; leyó The Logic of Modern Physics de Bridgman en esa época, además del Anticristo de Friedrich Nietzsche, y The Varieties of Religious Experience de William James; e incluso se dedicó a estudiar los Principia Mathematica de Bertrand Russell y Alfred N. Whitehead, que compró con cierto fervor ${ }^{47}$. En 1945, al acabar la guerra, Kuhn se matriculó en un par de cursos de lógica y metafisica con Henry M. Sheffer y Donald C. Williams, y de nuevo probó suerte oficialmente con la filosofía de la ciencia.

En esta segunda fase del interés de Kuhn por la filosofía hay indicios de un cambio que, aunque en su caso fue progresivo, constituyó un paso que en la filosofía académica consolidada estaba costando dar ( $\mathrm{y}$ de hecho acabaría por ser un cambio

recomendaba el ingreso en sociedades y cualquier forma de actividad extra-académica como un modo de integración plena en la universidad. La guía hacía una encuesta entre los alumnos de primero del curso previo en la que se les preguntaba si habían ingresado en alguna sociedad o habían participado en alguna actividad fuera de las clases, y cuál habra sido el resultado. Del $85 \%$ de los que participaron en algo sólo el 3,1\% lo lamentó. De los 141 estudiantes que no lo hicieron, 90 admitían que había sido un error.

45. Para una descripción del entorno familiar de Kuhn previo a Harvard, cf. nuestros Pilares, loc. cit., cap. 1, apdos. 1-2 (pp. 15-52).

${ }^{46}$ Carta de Thomas S. Kuhn a Emma K. Fisher, 27 de julio de 1943, Thomas S. Kuhn Papers, loc. cit., caja 12, carpeta 33, p. 3.

47 Ibid., p. 4. Sobre esas lecturas, cf. las fichas bibliográficas de Kuhn (clasificadas* cronológicamente) de esos títulos en Thomas S. Kuhn Papers, loc. cit., caja 9. 
de enfoque en toda regla). Kuhn ya avisaba en su carta a Fisher de julio de 1943 que se inclinaba a la filosofía "de la ciencia y por medio de ella" (cursivas mías). Los conceptos manejados en la ciencia, como tiempo, espacio o causa, eran completamente relevantes cuando se hacían reflexiones de tipo metafísico. Más aún cuando la corriente de la que provenían esas reflexiones, como por ejemplo la filosofía analítica británica de A. J. Ayer, B. Russell y G. E. Moore, trataba de prescindir de aquellos nombres de entidades que no poseyeran un referente reconocible. Pero para Kuhn ese empirismo logicista tendía a prescindir de demasiadas cosas debido sobre todo a una mala información sobre la naturaleza real de las teoría físicas.

Tal como Kuhn reflejó en uno de sus trabajos de clase en esta etapa, las teorías físicas no emplean, por ejemplo, una única forma de conexión causal. Russell, por ejemplo, asumía que la percepción de dos objetos de nuestra experiencia, $e_{1}$ y $e_{2}$, tal que a la percepción de $e_{1}$ le sucediera la de $e_{2}$ una vez transcurrido un tiempo $t$, permitía desarrollar leyes físicas como las de Newton. Éste era para Russell el esquema ontológico mínimo (y no trivial) para el concepto de causa ${ }^{48}$. Para Kuhn, el esquema de Russell se ajustaba a lo que esperaría un newtoniano de una noción de conexión causal. Pero para Kuhn la teoría física dice algo más que quizá sólo se capta desde fuera de ese punto de vista dinámico. Pongamos que $\mathrm{e}_{1}$ y $\mathrm{e}_{2}$ son las respectivas posiciones de una partícula cuyo movimiento se describe correctamente mediante las leyes de Newton. Una vez conocidas la posición, la velocidad y la aceleración de la partícula para $e_{1}$ (y conocido $\left.t\right), e_{2}$ queda determinado. Sin embargo, posición, velocidad y aceleración son propiedades dinámicas de un objeto que no agotan todas las posibles. De hecho, otras teorías no-newtonianas pueden ayudarnos a añadir nuevas propiedades dinámicas a esas tres, y una vez hecho esto la trayectoria de una partícula en un sistema dinámico newtoniano se convertiría meramente en un caso especial. Lo que nos indica Kuhn con esto es que el desarrollo de las leyes en una teoría física, incluyendo la de Newton, presupone condiciones que exceden la concomitancia de sucesos con el intervalo finito de tiempo obligado. Tales condiciones son parte integrante de cada teoría física, aunque, como en el caso de la de Newton, desaparezcan de nuestra vista en la presentación común de sus leyes y teoremas ${ }^{49}$.

48 Bertrand RuSSELL, "On the Notion of Cause", en Mysticism and Logic and Other Essays, Londres: George Allen \& Unwin, 1917 (orig. 1912), pp. 180-208, esp. p. 183. Siempre hay algún lapsos de tiempo entre causa y efecto, dice Russell, ya que no hay intervalos de tiempo infinitesimales (ibid., p. 187).

49 En concreto, nada en la presentación de manual de las leyes de Newton hace explícita la necesidad de la continuidad del espacio, del tiempo y de la trayectoria de la partícula sometida a 
Conviene destacar tres ideas principales de esta segunda perspectiva de Kuhn. Para empezar, es engañoso equiparar los objetos físicos a los del sentido común. Kuhn parece seguir (ni literal ni explícitamente, desde luego) lo que veíamos en Kemble: la física y el sentido común no se dan necesariamente la mano - - e incluso a veces la primera proporciona problemas al segundo- $y$ sobre todo la barrera entre ambos mundos existe, aunque no podamos verla. Para Kuhn, los filósofos han de ser conscientes de ella cuando tratan de aislar conceptos en su versión más general y abstracta (como hace Russell con la idea de causa). Esta perspectiva de Kuhn se resume bien en lo que indicaba a su tía Emma Fisher: su filosofía no sólo era de la ciencia, sino también a través de ella.

En segundo lugar está una idea que sirve de fundamento a la primera: para Kuhn la teoría física no es un conjunto tan unificado como parece que implica el uso simultáneo de términos idénticos en diferentes disciplinas y subdisciplinas. Las ideas de "causa" manejadas en la segunda ley de Newton y en la ley de Boyle, dice Kuhn, se diferencian en que en la segunda el lapso de tiempo que es crucial en la primera no tiene cabida. Aunque la relación entre eventos y conducta causal es diferente en ambos casos, podemos hablar de conexión causal en los dos ${ }^{50}$. Bridgman ya avisaba de ello cuando proponía su análisis operacional para la aclaración y en ciertos casos incluso la eliminación de aparentes sinónimos en térmi-

dichas leyes. Kuhn desarrolla todo este argumento en un trabajo para Donald C. Williams titulado "An Analysis of Causal Connexity", primer cuatrimestre del curso 1945/46, Thomas S. Kuhn Papers, loc. cit., caja 1, carpeta 3, esp. pp. 6-13. Para una descripción más amplia de ese trabajo, cf. MAYORAL, Pilares, loc. cit., cap. 2, apdo. 3.III (pp. 166-184).

${ }^{50} \mathrm{KUHN}$, "An Analysis of Causal Connexity", loc. cit., pp. 14-17. En la ley de los gases de Boyle (al menos para un gas ideal), si doblamos el volumen de un gas a temperatura constante, reduciremos su presión a la mitad. (Esto se suele expresar diciendo que $p V=c t e$.) Aquí, dice Kuhn, "(...) al cambio de volumen se le da una prioridad aparente en el tiempo y se convierte en pseudocausa. Sin embargo, no hay posibilidad de separación temporal de los diversos aspectos (en este caso presión, volumen y temperatura) que definen el estado físico de la sustancia. (...) El volumen y la presión del gas, por ejemplo, están inextricablemente unidos como aspectos diferentes de un sólo evento y no es posible que ninguno de ellos pueda ser determinado sin que el otro quede determinado y sea determinablem. (Ibid., pp. 15-16. Cursivas añadidas por nosotros.) Queda claro, sobre todo en la expresión más arriba subrayada, que cada noción de causa en cada parte de la física va ligada a una prefiguración -no necesariamente explícita en la teoría- de lo que podriamos llamar "evento mínimo" o "evento fundamental». Para poder reducir la noción de causa a una definición lo suficientemente general primero debemos saber cómo figuran esas entidades en cada teoría cientifica; y en segundo lugar hemos de poder controlar su variabilidad. De momento, nos indica Kuhn, nuestra comprensión de la idea de causa ha de continuar siendo todo lo vaga que ya era para Hume. (Ibid., p. 21.) 
nos que en realidad encierran significados distintos. La sinonimia, como ya dijimos, es en sí mismo un problema que el físico (y probablemente también el filósofo) ha de resolver.

Finalmente está la idea de que para Kuhn las teorías físicas no sólo surgen a partir de los datos de medición, de las observaciones, su clasificación y su pulido. La organización de una teoría física requiere la presencia de los vínculos psicológicos de los que tanto hemos hablado, los conceptos, que suelen ser muy difíciles de especificar, muy complicados de definir, de unificar y, en consecuencia, de reducir a los puros "enunciados de observación" de molde empirista (un antiguo proyecto positivista). Los conceptos no suelen aparecer definidos en las expresiones fundamentales de una teoría, pero para Kuhn (al igual que para Conant) sí cumplen un papel crucial y no sólo en la organización de una teoría sino también en su desarrollo.

Sólo en la última de las consecuencias, Kuhn se desvincula de manera ostensible de lo que cuenta Bridgman en The Logic of Modern Physics. Esto no debe sorprendernos, porque la lectura de ese libro le había dejado con la impresión de que la "filosofía operacional" (como Kuhn la llamó) no llegaba todo lo lejos que debía con Bridgman. Bridgman, a su juicio, "al final fracasa[ba] física y psicológicamente al no llegar lo suficientemente lejos en ninguna de ambas direcciones" , y añadía que «no [era] siempre consistente con la fil[osofía] operacional. Véase el uso de concepto" ${ }^{51}$. Sobre qué era para Kuhn "llegar lo suficientemente lejos física y psicológicamente" sólo podemos hacer conjeturas; pero al menos podemos aventurar lo siguiente. En lo referente a la línea "física", advertimos una crítica a Bridgman que sólo aparecerá unos ocho años después de la que acabamos de ver (de 1943). En 1951, Kuhn achacará a Bridgman inexactitud en el criterio operacional. Para Kuhn la medición de la longitud dentro de un mismo grupo de operaciones no deja de estar sometida a variaciones en torno a materiales y procesos. Bridgman no aporta un criterio unificador a este respecto ${ }^{52}$.

51 Thomas S. Kuhn, ficha de lectura de The Logic of Modern Physics, 3 de septiembre de 1943, Thomas S. Kuhn Papers, loc. cit., caja 9.

52 Thomas S. KUHN, The Quest for Physical Theory: Problems in the Methodology of Scientific Research, Conferencias Lowell, Boston Public Library, 3-30 de marzo de 1951, Thomas S. Kuhn Papers, loc. cit., caja 11, carpeta 33, conferencia 7, pp. 7-4 a 7-5. Kuhn, no obstante, no parece haber tenido del todo claro que la filosofía operacional pudiera consumar sus posibilidades. Retrospectivamente, Kuhn pensaba en 1965: «Tengo la impresión (...) de que me gustaba mucho 
En lo que se refiere a la línea "psicológica", podemos volver a la tercera de las consecuencias antes extraídas de la nueva perspectiva filosófica de Kuhn en 1945. Para Kuhn, los conceptos poseen una cierta autonomía en lo que respecta a su desarrollo. Conant también era consciente de esto y, como vimos, Bridgman mismo admitía que el cambio conceptual siempre era difícil. Los conceptos formaban una red coherente difícil de alterar, aunque era necesario hacerlo cuando los datos lo pedían. Los conceptos estaban a su servicio. Pero para Kuhn había algo más que decir sobre esa "servidumbre conceptual". El carácter auxiliar de los conceptos y la aplicabilidad de un análisis operacional como aclaración de su verdadero significado físico, algo admisible para Kuhn, no implicaba a la par que los conceptos no tuvieran una importancia mayor para la teoría física de la que Bridgman, Conant y el resto de la filosofía de la ciencia les estaba concediendo, e incluso una mayor autonomía. "Papel crucial de los conceptos en el desarrollo de la teoría física" y "algo de autonomía para ellos" son dos ingredientes peligrosos de manejar para completar una visión de la ciencia como investigación de la realidad, pero poco a poco, a partir de 1945, Kuhn aprendió a conjugarlos. Veamos cómo.

\section{Kuhn, Koyré y la complejidad de la ciencia}

Thomas S. Kuhn conoció la historia de la física de la mano de Conant, pero no siguió su mismo esquema histórico; o al menos no del todo. Cuando Conant le invitó a colaborar con él en 1947, Kuhn ya buscaba consolidar la perspectiva que hemos visto en el apartado anterior. De hecho, en mayo de 1947 (i.e., antes de empezar a trabajar con la historia de la ciencia por primera vez) Kuhn se presentó en la primera reunión de los docentes del programa de Conant con un esquema muy detallado de los contenidos generales, desglosados desde un punto de vista filosófico (según sus palabras), de un curso de educación general en ciencias físicas ${ }^{53}$. En ese esquema destaca el papel al que relega el proceso de inducción, fundamental en la metodología empirista. Los objetos de los que se ocupa la ciencia -que, como diji-

la perspectiva general de Bridgman pero no estaba seguro del todo de que pudiera llevarla hasta donde él quería. (...) Creo que era un poco escéptico con el operacionalismo". Y sobre los análisis operacionales concretos recordaba: "(...) vi varios de sus análisis especificos (en particular, creo, el análisis de la distancia y el espacio) algo ingenuos». Y Kuhn remataba diciendo: «No estaba claro para mí que el trabajo no pudiera hacerse, pero no creía que pudiera hacerse tan fácilmentew. Carta de Kuhn a Robinson, 8 de marzo de 1965, loc. cit., p. 1.

53 Thomas S. KuHN, "Objectives of a General Education Course in the Physical Sciences", mayo de 1947, Thomas S. Kuhn Papers, loc. cit., caja 1, carpeta 1. 
mos más arriba, no eran para Kuhn los del sentido común - no son los puros objetos de nuestra percepción sino algo más elaborado. Las generalizaciones de la ciencia se hacen a partir de estos últimos. Tales objetos, que Kuhn denomina «objetos científicos" por oposición a los más primitivos, los de la percepción o "perceptivos", se clasifican a partir de la selección de una serie de rasgos considerados esenciales. Las generalizaciones se basan, en concreto, en esas clasificaciones. Más importante todavía es que las clasificaciones cambian y las generalizaciones también cambian con ellas. El cambio en las clasificaciones sigue un proceso que Kuhn denomina en ese esquema de 1947 de "abstracción sucesiva".

Así, la historia de la ciencia de Kuhn es en estos momentos el recorrido y cambio de ciertos objetos perceptuales reducidos a una serie de propiedades y transformados con ello en "objetos científicos». El mecanismo de abstracción sucesiva del que habla Kuhn requiere principalmente de un primer motor (en absoluto inmóvil): la mente humana. En ella se combina toda la información disponible mediante la manipulación lógica y matemática («simbólica», en general) de las generalizaciones y la purificación empírica de los rasgos de los objetos científicos. De esa mente parten las nuevas clasificaciones y surge toda nueva información. Tanto la experimentación como la observación controlada realizada en geología y en biología son formas de especificación con miras a la producción de definiciones. El experimento, dice Kuhn en ese esquema, es el "arquitecto" de la definición científica. El papel de arquitecto no tiene que ver con la filosofía operacional, dice Kuhn explícitamente, sino con la conexión "históricamente demostrada del experimento con los "objetos científicos". Por así decirlo, la investigación experimental, que el empirismo tradujo en origen de la teoría científica, es para Kuhn un instrumento que perfila la información perceptiva. El origen de la información y de la teoría está en la mano moldeadora de la mente humana.

Algunos años más tarde, Kuhn encontrará una justificación psicológica para reordenar en este sentido la base epistemológica del método científico, pero no vamos a entrar en eso ${ }^{54}$. Con esta perspectiva nos damos ya cuenta de por qué motivo Kuhn vio su perspectiva del desarrollo científico no sólo reflejada sino óptimamente materializada en la obra del ruso francófono y políglota Alexandre (Alejandro Vladímirovich) Koyré.

${ }^{54}$ Cf. Mayoral, Pilares, loc. cit., cap. 3, apdos. $2-3$ (pp. 275-324) y cap. 4, apdos. 3-5 (pp. 338-295). 
Para Koyré, la huella del intelecto está detrás de las transformaciones que causaron la Revolución Científica del siglo XVII. Es cierto, por un lado, que ese episodio histórico ha sido tratado, nos recuerda Koyré, como el inicio de una empresa de investigación (la ciencia moderna) que se caracteriza por "(...) su carácter empírico y concreto por oposición al carácter abstracto y libresco de la ciencia clásica y medieval. La observación y la experiencia llevando una ofensiva victoriosa contra la tradición y la autoridad: ésa es la imagen (...) que se nos da habitualmente de la revolución intelectual en el siglo XVII (...) $)^{55}$. Sin embargo, para Koyré, al destacar el rasgo empirista de esa ciencia podemos cometer el error de entender la investigación de los fenómenos del modo en que hizo Francis Bacon. Según Koyré, el progreso técnico del Renacimiento (siglos XV-XVI) en la construcción de máquinas (por ejemplo, en metalurgia, construcción naval, conducción hidrológica, etc.) hizo concluir a Francis Bacon y a sus seguidores que la pura especulación teórica se identificaba con el mencionado "carácter abstracto y librescon y que lo que la ciencia requería era una perspectiva más práctica, más cercana a los mecanismos observables; a aquello, en suma, de lo que podemos hablar por pura referencia ostensible. Así, para Bacon, según Koyré, «(...) la inteligencia debe limitarse al registro, a la clasificación y a poner en orden los hechos del sentido común (...)». La ciencia no es ni debe ser "(...) más que un resumen, generalización o prolongación del saber adquirido en la práctica (...) $)^{56}$.

Para Koyré, «Bacon jamás comprendió nada de la ciencia»" 57 :

Lo de "Bacon, iniciador de la ciencia moderna" es una broma, de muy mal gusto, que todavía repiten los manuales. En realidad, Bacon no comprendió nunca nada de la ciencia. Es crédulo y se halla totalmente desprovisto de espíritu crítico. Su mentalidad está más próxima a la alquimia, a la magia (cree en las «simpatías»), en pocas palabras, a la de un

5s Alexandre KoYre, "An Experiment in Measurement", Proceedings of the American Philosophical Society, 97 (2) (abr. 1953), pp. 222-237. Citamos por la traducción de E. Pérez Sedeño y E. Bustos en Alexandre KoYRE, "Un experimento de medición", en sus Estudios de historia del pensamiento cientifico, Madrid: Siglo XXI, 1990 (10ª ed.; orig. 1973), pp. 274-305, esp. p. 274.

${ }^{56}$ Alexandre KoYré, "Du monde de l' " a-peu-près » à l'univers de la précision”, Critique, 28 (1948). Citamos por la traducción de Antonio Beltrán en Alexandre KoYRE, "Del mundo del "aproximadamente" al universo de la precisión", en su Pensar la ciencia, introducción de Carlos Solís, Barcelona: Paidós, 1994, pp. 117-145, esp. pp. 123-124.

57 Ibidem. 
primitivo o un hombre del Renacimiento, que a la de un Galileo o incluso a la de un escolástico. ${ }^{58}$

Según Koyré, Bacon jamás comprendió la naturaleza intelectual del hilo rector de la experimentación y de la teoría científica, una mecánica mental comprendida y expresada por Descartes en la que el estudio (casi "disección») de los fenómenos en la experimentación conlleva una actividad del intelecto. Es decir, no una fascinación con la complejidad natural, fenoménica, sino una intervención en ella con el ánimo de reducirla a piezas (y relaciones entre ellas) que sean tanto reconocibles como manejables por el hombre ${ }^{59}$. La experiencia, nos recuerda Koyré, es una pregunta que se le hace a la naturaleza y algo así conlleva una intención, un proyecto de aclaración que presupone una teoría ${ }^{60}$.

La experiencia, como mera observación de los objetos cotidianos (i.e., la observación propia del "sentido común»), es para Koyré parte de la ciencia clásica y medieval. Como señalaba Émile Meyerson (de quien Koyré se hace eco), esa tendencia es más bien un obstáculo al avance de la ciencia. Así, por ejemplo, el principio de inercia es contrario al sentido común, porque la idea de que un cuerpo puesto en movimiento se empeña en (i.e., tiende a) moverse sin más, es decir, sin tratar de llegar a algún punto o lugar concreto, no es válida de inmediato a una mente (una mirada) no entrenada para considerarla una extrapolación verosímil del movimiento que solemos ver. Éste es finito; normalmente se limita a ser un recorrido entre dos puntos. A decir verdad, los filósofos griegos y los medievales no sólo no tuvieron ese principio en cuenta en su física, sino que ni siquiera pudieron tenerlo como un principio dinámico válido ${ }^{61}$. Y siguiendo a Paul Tannery y a Pie-

${ }^{58}$ Alexandre KOYRE, Etudes galiléennes, París: Hermann, 1940 (con fecha de 1939). Citamos por la traducción de Mariano González Ambóu en Alexandre KoYrÉ, Estudios galileanos, Madrid: Siglo XXI, 1990 ( $5^{2}$ ed.), p. 2, nota 6. El Bacon de Koyré es un erudito renacentista fascinado por la nueva maravilla de las capacidades técnicas y los nuevos descubrimientos que predica sobre ellos, no un científico. En términos de los que hablaremos más abajo, el Bacon de Koyré está afectado por la parte más negativa ("el reverso de la medalla", cf. infra) del Renacimiento. Como veremos, para Kuhn esto no reduce la importancia del papel de Bacon y del, así llamado, "baconianismo". Su principal discrepancia con Koyré irá por este camino, como veremos.

59 Cf. KOYRE, "Un experimento de medición", loc. cit., p. 275; KOYRE, "Del mundo del "aproximadamente" al universo de la precisión", loc. cit., p. 124.

${ }^{60}$ KOYRE, "Un experimento de medición", loc. cit., p. 275.

${ }^{61}$ Koyré cita Identité et realité de Émile MEYERSON (París, 1926, 3aed, esp. pp. 124 y 156) en su "Galileo and Plato", Journal of the History of Ideas, 4 (4) (oct. 1943), pp. 400-428. Citamos 
rre Duhem, Koyré nos recuerda que precisamente la física de Aristóteles y el tratamiento del movimiento local mediante el recurso dinámico del impetus por parte de Juan Buridán y Nicolás de Oresme están más cerca de la experiencia de sentido común que la física de Galileo o de la de Descartes ${ }^{62}$.

Este aparente galimatías se explica bien en un breve texto de Koyré titulado "La aportación científica del Renacimiento", una ponencia de 1949. Ahí, Koyré exhibe una frase que es crucial para entender el lazo que existe entre él y Kuhn (y del que parte esta perspectiva histórica compartida con otros filósofos e historiadores franceses como Tannery, Duhem y Meyerson): “(...) me parece», dice Koyré, "que en nuestro pensamiento lo posible prevalece siempre sobre lo real, y lo real no es más que el residuo de lo posible; se coloca o se encuentra en el marco de lo que no es imposiblen. (Cursivas añadidas.) $\mathrm{Al}$ aplicar esto al desarrollo histórico de la ciencia desde Aristóteles hasta Galileo y Descartes, continúa:

En el mundo de la ontología aristotélica hay una infinidad de cosas que no son posibles, una infinidad de cosas, pues, que se sabe de antemano que son falsas.

Una vez que esta ontología es destruida y antes de que una nueva, que no se elabora hasta el siglo XVII, haya sido establecida, no hay ningún criterio que permita decidir si la información que se recibe de tal o cual whecho» es verdadera o no. (...) Ahora bien, por el hecho mismo de la destrucción de la ontología medieval, de la ontología aristotélica, el Renacimiento se ha encontrado lanzado o reducido a una ontología mágica, cuya inspiración se encuentra por todas partes. (...) De este modo, si se quisiera resumir en una frase la mentalidad del Renacimiento, yo propondría la fórmula: todo es posible. (...) Ahora bien, si esta credulidad de «todo es posible» es el reverso de la medalla, hay también un anverso. Este anverso es la curiosidad sin límites, la agudeza de visión y el espíritu de aventura que llevan a los grandes viajes de descubrimientos y a las grandes obras de descripción. ${ }^{63}$

por la traducción de E. Pérez Sedeño y E. Bustos en Alexandre KOYRE, "Galileo y Platón", en sus Estudios de historia del pensamiento cientifico, loc. cit., pp. 150-179, esp. pp. 152 (nota 8) y 154 (nota 11).

${ }^{63}$ Koyré cita en este caso a Pierre DuHEM y su Le système du monde (Paris, 1913, vol. 1, esp. pp. 194 y sigs.); ibid., p. 152, nota 9. 
Para Koyré, la Revolución Científica del siglo XVII se sirve del campo ontológico ahora allanado que le proporciona el Renacimiento. En él, ya no rige del todo Aristóteles (aunque tampoco ha desaparecido, ni siquiera de la primera obra dinámica de Galileo) con su acotación y jerarquía cosmológicas y ontológicas. Al menos, ahora hay lugar para otras perspectivas, para otras tradiciones y, por ejemplo, para el redescubrimiento de Arquímedes, crucial en el desarrollo de la nueva física de Galileo ${ }^{64}$. Concretamente, sobre cimientos prioritariamente intelectuales (en especial con la aplicación de la geometría a la representación y el estudio del espacio, el cosmos y el movimiento no sólo celeste sino también terrestre, local) se forja la nueva ciencia de Galileo y de Descartes ${ }^{65}$. Ésta para Koyré, y a diferencia de Bacon, no es hija de la técnica, de la experiencia artesanal acumulada. Para Koyré, Galileo y Descartes no trabajan a partir de la simple extrapolación teórica de la ingeniería y artesanía renacentista, sino desde la nueva perspectiva matemática del mundo físico ${ }^{66}$.

Koyré no niega que el experimento cumpla también su función, ni que ésta sea en ciertos momentos incluso crucial. Pero para Koyré esa función se ajusta a las exigencias de la teoría. Así, Koyré muestra en otro de sus textos fundamentales, From the Closed World to the Infinite Universe (1957), que la presencia del telescopio no modificó en lo esencial el argumento de Kepler a favor de la finitud del universo. La lectura del Sidereus nuncius de Galileo no supuso para Kepler el abrazo de las tesis infinitistas de Giordano Bruno, sino una reafirmación en su tesis de que una exploración racional a partir de la evidencia astronómica (de los fenómenos astronómicos) deja claro lo que ya antes mantenía: que el universo es finito ${ }^{67}$. El instrumento científico es para Koyré un fiel sirviente de las ideas, no el dictador de teorías que afirma Bacon.

${ }^{64}$ Ibid., p. 44. Cf. J. V. MAYORAL, "Creencias y soluciones: Galileo y el problema de la aceleración", Éndoxa, 11 (1999), pp. 101-145, esp. pp. 116-124 y 126-134.

${ }^{65}$ KOYRÉ, "Galileo y Platón", loc. cit., p. 154.

${ }^{66}$ Ibid., pp. 151-152. Para una versión más completa de toda esta perspectiva, cf. sus Etudes galiléennes, loc. cit., esp. el primer estudio, apdo. IV ("Galileo»).

${ }^{67}$ Alexandre KoYRE, Del mundo cerrado al universo infinito, trad. Carlos Solís, Madrid: Siglo XXI, 1999 (4a ed.), esp. cap. III, pp. 64-76. Para la traducción al castellano del Sidereus nuncius de Galileo y de la Dissertatio cum nuncio Sidereo de Kepler (entre otros documentos), cf. Carlos Solís (ed.), Galileo-Kepler: El mensaje y el mensajero sideral, Madrid: Alianza, 1990 (orig. 1984). 
Kuhn se vio seducido por la perspectiva koyreana de la ciencia. Entre mayo de 1947 y el final del primer cuatrimestre del curso siguiente (1947/48), cuando se dedicó en cuerpo y alma a las clases sobre historia de la dinámica encomendadas por Conant, Kuhn se sumergió en la historia de la ciencia por primera vez; ahí conoció la obra de Koyré y leyó sus Études galiléennes ${ }^{68}$ :

(...) a sugerencia de Bernard Cohen leí los Études galiléennes de Koyré. Me encantaron. Quiero decir que el libro me enseńó un modo de hacer las cosas que yo nunca había imaginado. En un sentido no era tan extraño como podría parecer, porque había leído y admirado mucho el libro de Lovejoy, Great Chain of Being. Pero que se pudiera hacer eso con la ciencia era algo que no se me había ocurrido, y esto es lo que, en algún sentido, Koyré me enseñó. Y fue importante. ${ }^{69}$

Kuhn, en efecto, conocía de sus estudios literarios en Harvard una perspectiva del estudio de las obras intelectuales del pasado que se basaba en la investigación contextual de los fundamentos del pensamiento y la creación. Esta perspectiva se oponía por principio al idealismo de George Santayana y sus ideales estéticos equiparables a ideas platónicas absolutas y atemporales. La nueva perspectiva presuponía que nuestro canon estético contemporáneo podía ocultar el objetivo y el significado real (i.e., original) de la obra artística y literaria del pasado. Esta perspectiva permitía comprender, por ejemplo, los "fracasos" (posiblemente aparentes por lo tanto) de las grandes figuras de la historia de las letras, de John Donne a William Shakespeare. La perspectiva de Koyré era muy semejante, pero se aplicaba al desarrollo de la ciencia.

En Koyré, Kuhn encontraba el ingrediente que la historia operacional de Conant no podía aportar. En cierto modo, Conant daba entrada al sujeto como agente creador de la ciencia. Y no en el sentido de Bacon y Pearson, es decir, no

${ }^{68}$ Kuhn se ocupó de una serie de clases que Conant dejaba en su curso para casos históricos de física que completaban un programa principalmente dedicado a la historia de la química, de Boyle a Dalton. Para ello, Kuhn solicitó una excedencia de la beca del National Research Council, ya que ésta le impedía dedicar tiempo a otra actividad académica que no fuera su propia investigación de física del estado sólido. La excedencia le fue concedida hasta el primero de febrero de 1948. Cf. la carta de W. Albert Noyes (presidente del National Research Council) a Thomas S. Kuhn, 12 de junio de 1947, Thomas S. Kuhn Papers, loc. cit., caja 14, carpeta 17.

${ }^{69}$ BALTAS et al., op. cit., pp. 332-333. 
como un mero procesador de datos que no pone nada de sí mismo en sus descripciones y explicaciones. El agente creador de Conant diseñaba su procedimiento experimental para intervenir en los fenómenos y aprender algo nuevo de ellos. Sin embargo, Conant no iba más allá, y aquí cabe una cierta crítica por nuestra parte (seamos o no Thomas S. Kuhn). Si el individuo elegía la partición de la realidad, la clasificación de la complejidad fenoménica, ¿en qué medida se sostiene que los conceptos meramente "sirvan" a los datos? Es decir, la jerarquía de que son los datos los que legislan el uso conceptual, ¿no resulta una pretensión ingenua? (Y cabría decir que fatua y caciquil, pues presupone una jerarquía ontológica que da, sin más, toda la capacidad de decisión a algo fuera de nuestra voluntad.) Conant no hablaba de nada al respecto. Como ya hemos dicho, mantenía la misma perspectiva operacional de Bridgman, que obliga a evitar la presunción de sinonimia terminológica (i.e., una supuesta identidad conceptual) cuando no existe una prueba empírica de la misma.

Kuhn en cambio, desde el principio (i.e., mayo de 1947), sí veía una alternativa a la jerarquía empirista de Bridgman y Conant. Para él, los objetos manejados por la ciencia (los objetos cientificos) nacen de la mente humana, no sólo ni en principio de los datos. Más aún, son estos últimos los que sirven a un orden clasificatorio establecido y sólo se obtienen a expensas de las clasificaciones y objetos disponibles ${ }^{70}$. Así, los objetos científicos son los únicos contactos con la realidad de que dispone la ciencia; son la materia en que se moldea el conocimiento científico. Por este motivo, si empleáramos el procedimiento operacional para eliminar conceptos (o "unidades conceptuales" que nos permiten establecer enlaces abstractos) porque no conocemos el modo de demostrarlos empíricamente, negamos por principio un modo de representación como mínimo potencialmente valioso, ya que o bien tal enlace conceptual, o bien su sustituto, pueden ser uno de los dos reales. En su ausencia es dificil saber si alguna vez llegaremos a explorar esa vía.

Lo que Koyré muestra es precisamente que la ciencia cuando tiene éxito no actúa como dice Bridgman, sino de un modo más parecido al señalado ya entonces por Kuhn. En la frase que hemos reproducido en cursiva más arriba (pues nos parece aforística y representativa de una perspectiva nueva de la naturaleza de la ciencia), Koyré decía que en nuestro pensamiento lo posible

\footnotetext{
${ }^{70}$ KUHN, "Objectives of a General Education Course in the Physical Sciences", loc. cit.
} 
prevalece siempre sobre lo real y esto último, lo real, que no es más que el residuo de lo posible; se halla en el marco de lo que no es imposible. Dicho de otro modo, nuestra representación teórica de la realidad bebe de una fuente que excede lo que podemos probar y entra dentro de la conjetura (i.e., de lo que podria llegar a darse). El Galileo que Koyré nos presenta, su Descartes, su Kepler, trabajan acorde a esta premisa sobre el pensamiento humano. En la figura del Galileo de Koyré, Kuhn podía encontrar, y de hecho encontró, la manifestación más acabada de sus propias ideas sobre la naturaleza de la ciencia. Gracias a Koyré, Kuhn se permitió ahondar en las ideas que ya llevaba consigo, y éstas lo condujeron incluso más allá de Koyré, especialmente en lo referente al papel de la tradición baconiana y a la función de los instrumentos en el desarrollo de la física.

Pero antes de pasar a ello hemos de finalizar nuestra idea de la diferencia Conant-Kuhn tal como queda a partir de lo dicho. En la perspectiva de Conant está claro que hay un mandato de la operación experimental y que, bajo el escenario conceptual, auxiliar y cambiante, se acumula un conocimiento empírico de la realidad. De J. Priestley a A. Lavoisier puede haber una reducción de la exactitud en un sentido, ya que, nos recuerda Conant, la aplicación de Lavoisier de un principio contable para estudiar la reacción química conlleva pasar por alto errores cuantitativos en ocasiones difíciles de admitir - y que Priestley, por ejemplo, no aceptaba- Sin embargo, ese principio contable de Lavoisier le permitía especificar mejor el proceso de reacción, sus "entradas" (reactivos) y sus "salidas" (productos), cuantificarlo con exactitud y hacer de la química una ciencia de la medida. Con ese paso enriquecemos nuestro conocimiento del detalle cuantitativo que subyace a los procesos químicos: Así, acumulamos información.

Pero Kuhn considera que Lavoisier no hace mejor el trabajo de Priestley, como Newton no hace mejor el de Aristóteles. No podemos decir que haya acumulación, por tanto. Como Kuhn le decía a Conant:

Abrías On Understanding Science discutiendo la acumulación como el rasgo distintivo de la ciencia. Luego me mandaste mirar la dinámica prenewtoniana. Volví de esa misión convencido de que la ciencia no era acumulativa en el sentido más importante. Newton no trataba de hacer mejor el trabajo de Aristóteles; más bien Aristóteles había estado tratando de hacer un 
trabajo diferente y uno que Newton no hizo tan bien. ¿Dirías que la artesanía fue meramente un modo menos eficiente de hacer lo que el sistema industrial hizo más tarde? ${ }^{71}$

La acumulación de Conant y la eliminación bridgmaniana de los upseudo-sinónimos" terminológicos van de la mano. Esta segunda permite conocer nuevas relaciones entre resultados de medición ( $y$ obtener otros) de un modo que previamente pasaba desapercibido. Para Kuhn, hay sin embargo intenciones, disposiciones, cuya prioridad psicológica y teórica en la ciencia hace que no podamos hablar tan a la ligera de acumulación y que, en su lugar, tengamos que prestar atención (como hacía Koyré) a las ideas, a los conceptos. Sólo éstos nos permiten decir por qué vía se desarrolla la ciencia y en qué medida acumulamos (o no) información, conocimiento.

\section{Kuhn y Koyré: La divergencia}

A partir del final del primer curso junto a Conant (primer cuatrimestre de 1947/48) y después de dar fin a su investigación doctoral (hacia el final de 1948), Kuhn dedicó casi por entero los casi tres años siguientes (1949-1951) como fellow de la Society of Fellows de Harvard a formarse lo suficiente como historiador de la ciencia para dar las clases del curso de educación general. De paso, Kuhn buscó moldear con más detalle y fortalecer las opiniones que acabamos de ver sobre los fundamentos del desarrollo de las ciencias físicas.

Ya que estos fundamentos eran conceptuales, Kuhn buscó un asiento filosófico para esa reordenación metodológica. Es decir, algo parecido a una versión epistemológica sólida que subyaciera a ese desarrollo típicamente koyreano. Así, Kuhn abrazó con entusiasmo las directrices provenientes de la epistemología empirista de Jean Piaget; su enfoque en la evolución mental del sujeto desde niño; su visión de las categorías mentales como fragmentaciones y clasificaciones completas del continuo de la percepción, que poco a poco, a medida que el individuo se vuelve adulto, se vuelven más específicas y más complejas. Para Kuhn, Piaget

${ }^{71}$ Carta de Thomas S. Kuhn a James B. Conant, 29 de julio de 1961, Thomas S. Kuhn Papers, loc. cit., caja 25, carpeta 53, p. 5. Hemos traducido bome industry en el original por "artesanía" (cabría también quizá «industria doméstica", por ejemplo). La cuestión es la oposición a la línea de trabajo sistemática de la industria moderna. 
daba con el quid de la cuestión al mostrar que la mente evoluciona no por el aumento del bagaje de datos acumulados, sino por el de la complejidad del aparato categórico del individuo. La tendencia rectora es a lograr una disminución de la auto-contradicción mediante la anulación del uso ambiguo de los conceptos (algo en lo que la interacción social tiene mucho que ver como estímulo causal $)^{72}$.

Para Kuhn la evolución de la física se entendía mucho mejor bajo este prisma piagetiano. El propio Koyré lo había captado así y hablaba de dicha evolución como de una transición a una ontología más abstracta a partir de otra muy sujeta a las apariencias inmediatas, al estado primigenio (i.e., de sentido común) de la familiaridad del individuo con los fenómenos naturales. Para Koyré, el Cosmos aristotélico era un entorno reducido, cuasi-doméstico, en el que los estados tenían valor ontológico en sí mismos y no eran consecuencias de un orden subyacente más complejo. Un rasgo crucial de la Revolución Científica del siglo XVII había sido la ruptura con este Cosmos finito ${ }^{73}$. Bajo esta perspectiva koyreana, el recorrido de Galileo y Kepler a Descartes y Newton era fruto del esfuerzo por fundar una nueva física alternativa a la aristotélica - más aún, una mecánica terrestre y celeste unificada, algo realmente nuevo- que explicase el cambio (i.e., los fenómenos naturales) en un universo ahora infinito y ya no jerarquizado. En sus palabras, la Revolución Científica del siglo XVII se puede describir «(...) diciendo que conlleva la destrucción del Cosmos; es decir, la desaparición, en el campo de los conceptos filosófica y cientificamente válidos, de la concepción del mundo como un todo finito, cerrado y jerárquicamente ordenado (...). Ese Cosmos se ve sustituido por un universo indefinido y aun infinito que se mantiene unido por la identidad de sus leyes y componentes fundamentales y en el cual todos esos componentes están situados en un mismo nivel del ser ${ }^{74}$. Koyré reconoció el paralelismo con Piaget ante el propio Kuhn: «Recuerdo de manera vívida», decía Kuhn,

72 En la primera época en la Society of Fellows, Kuhn leyó las traducciones al inglés de dos libros de Jean PiageT, Le jugement et le raisonnement chez l'enfant (Neuchâtel: Delachaux et Niestlé, 1924) y Les notions de mouvement et de vitesse chez l'enfant (Paris: Presses Universitaires de France, 1946). Más adelante se introduciría en otro texto de Jean PLAGET, La causalité physique chez l'enfant (París, Alcan, 1927). Para la información cronológica de las dos primeras lecturas y las notas de Kuhn sobre ellas, cf. Thomas S. KUHN, Handy Notebook, 31 de marzo-6 de julio de 1949, Thomas S. Kuhn Papers, loc. cit., caja 1, carpeta 7, esp. pp. 9-22.

${ }^{73} \mathrm{El}$ otro rasgo fue, según Koyré, la geometrización del espacio. Cf. KoYRE, "Galileo y Platón", loc. cit., p. 154.

${ }^{74}$ KOYRE, Del mundo cerrado al universo infinito, loc. cit., p. 6. 
"que esa influencia [de Piaget] apareció en mi primer encuentro con Alexandre Koyré (...). Le dije que había aprendido a comprender la física de Aristóteles a partir de los niños de Piaget. Su respuesta (...) [fue] que era la física de Aristóteles la que le había enseñado a él a entender a los niños de Piaget (...) ${ }^{75}$.

Para Kuhn, este paralelismo era sumamente interesante y no dejó de explorarlo. Al término de su periodo en la Society of Fellows, Kuhn exhibió públicamente (por primera vez en su vida) sus ideas al respecto con motivo de las conferencias anuales del Instituto Lowell, que su presidente, Ralph Lowell, le había invitado a pronunciar (un privilegio con frecuencia concedido a los jóvenes junior fellows de Harvard). El contenido de estas conferencias es tan extenso que carece de sentido intentar describirlo en pocas páginas ${ }^{76}$. Pero sí podemos resumir brevemente la parte de ellas que refleja ese paralelismo.

Kuhn dedicó mucho espacio de las dos primeras conferencias (de un total de ocho) a describir las claves de la revolución galileana en el estudio del movimiento. Siguiendo muy de cerca a Koyré, Kuhn situaba el origen de esa revolución en un "cambio de problema», como él lo llamaba ${ }^{77}$. A partir de Aristóteles, los escolásticos de París (J. Buridán y N. Oresme) habían logrado convertir el movimiento en una propiedad de los objetos, ya no un avatar accidental de su estado como en la física aristotélica ${ }^{78}$. El cambio de posición pasaba a convertirse así en un objeto científico; un objeto evolucionado a partir de la pura percepción, con rasgos particulares a aislar y (como más tarde mostraría Galileo) con una conducta sencilla de establecer en términos de lo que hoy conocemos como "ley física" o "ley natural». Los parisinos, al igual que los calculatores del Merton College de Oxford, habían esbozado muchos de esos rasgos del movimiento, e incluso habían dado con métodos matemáticos (geométricos por parte de Oresme y con el llamado «álgebra de palabras» por parte

75 Thomas S. KUHN, "Concepts of Cause in the Development of Physics", Etudes d'épistémologie génétique, 25 (1971), pp. 7-18. Citamos por la reedición en Thomas S. KuHN, The Essential Tension: Selected Studies in Scientific Tradition and Change, Chicago: The University of Chicago Press, 1977, cap. 2 (pp. 21-30), pp. 21-22.

${ }^{76}$ Un estudio independiente se dedicará a ello. Entre tanto, cf. nuestros Pilares, loc. cit., cap. 4 (pp. 325-397).

77 Cf. KOYRE, Estudios galileanos, loc. cit.; KOYRE, "Un experimento de medición", loc. cit.; y Alexandre KOYRE, "Galilée et l'experience de Pise: à propos d'une légende", Annales de l'Université de Paris, 12 (1937), pp. 441-453.

${ }^{78} \mathrm{KuHN}$, The Quest for Physical Theory, loc. cit., conferencia 2, p. 13. 
de los oxonienses mertonianos) para describirlo ${ }^{79}$. El movimiento se podría reducir a una serie de rasgos cuantitativos ${ }^{80}$.

Para Kuhn, Galileo había aprovechado esos pasos con un objetivo algo diferente en mente al de estos filósofos medievales. Él no deseaba corregir las categorías lógicas de Aristóteles, sino desarrollar la física con un vocabulario geométrico (arquimediano) y para un Cosmos ya no geocéntrico, sino copernicano. Galileo, repetía Kuhn, no se había servido de ningún nuevo hecho científico. La evidencia empírica de que disponía no era el fruto de una «nueva experimentación controlada». En su mayor parte, nacía de una observación de los fenómenos que ya estaba al alcance de los filósofos en la época de Aristóteles. No había por qué negar que Galileo hubiera explorado con experimentos como el del plano inclinado el mundo de los fenómenos. (Un "experimento» que, para Koyré, recibe mejor la denominación de "experiencia»" ${ }^{81}$.) Pero el papel del experimento no era, ni para Kuhn ni para Koyré, el de explorar un terreno desconocido - como en la perspectiva de F. Bacon-. Como Kuhn señalaría en un conocido artículo años después, «El camino que lleva de la ley científica a la medición científica rara vez se puede recorrer en la dirección inversa» ${ }^{82}$.

En sus Conferencias Lowell, Kuhn describía con detalle el ejemplo de Galileo porque le servía para ilustrar el mecanismo del desarrollo científico. El mecanismo generaba un ciclo discontinuo de desarrollo científico como el que luego aparecería en Structure. Kuhn introducía por primera vez el conocido ciclo en tres pasos: (1) ciencia normal (aquí "periodo clásico» ); (2) periodo de crisis; y (3) revolución científica ${ }^{83}$. El mecanismo generador del ciclo se basaba en un prisma piagetiano de la mente humana extrapolado a la propia teoría científica. Para

${ }^{79}$ Ibid., conferencia 2, pp. 21-22.

${ }^{80}$ Para este cambio, cf. A. C. CROMBIE, Historia de la ciencia: De San Agustin a Galileo, 2. La Ciencia en la Baja Edad Media y comienzos de la Edad Moderna: siglos XIII al XVII, trad. José Bernia y Luis García Ballester, Madrid: Alianza, 1974 (orig. 1959), pp. 86-93. Cf. también Marshall CLAGETT, The Science of Mechanics in the Middle Ages, Madison (Wis.): The University of Wisconsin Press, 1959, pp. 519-540.

${ }^{81}$ Para esa distinción, cf. KOYRE, Estudios galileanos, loc. cit., p. 3 y 144-147; KOYRE, "Galileo y Platón», loc. cit., pp. 152-153.

82 Thomas S. KUHN, "The Function of Measurement in Modern Physical Science", Isis, 52 (1961), pp. 161-193. Citamos por la reedición en KUHN, Essential Tension, loc. cit., cap. 8 (pp. 178-224), p. 219.

${ }^{83}$ Kunn, The Quest for Physical Theory, loc. cit., conferencia 5, pp. 3a-2 y sigs. 
Kuhn, la teoría es algo más que un conjunto de enunciados verificables y lógicamente conectados; algo más próximo a las ideas científicas y filosóficas de Koyré, de las que dependía el desarrollo de la ciencia y, en especial la Revolución Científica del siglo XVII ${ }^{84}$. Kuhn denomina al conjunto filosófico-científico "orientación científica". Para Kuhn, siempre existe una orientación detrás del trabajo del científico; una única orientación ${ }^{85}$. Desde un punto de vista psicológico y epistemológico, no hay otro modo de practicar la ciencia (como no lo hay de enfrentarse cotidianamente al mundo que nos rodea). Así pues, como la mente del individuo de Piaget, la teoría científica no cambia sencillamente al englobar más hechos en su seno, sino al distribuir mejor lo conocido e incluir también lo inadvertido previamente; es decir, cambiando la clasificación y elaborando los objetos científicos. Es lo que se refleja precisamente en el caso de Galileo: la transformación revolucionaria procede de un cambio de problema; de un nuevo enfoque con otros objetivos y medios. En suma, de una nueva orientación.

Desde 1951, Kuhn se embarcó en una autoformación más sólida como historiador de la ciencia y hasta 1959 no volvió a ocuparse de los problemas de los que hablaba en las Conferencias Lowell. El motor de esta dedicación a la historia de la ciencia es práctico. Kuhn logró sus primeros puestos docentes en Harvard, y más tarde en Berkeley, como profesor de historia de la ciencia. Las exigencias de la búsqueda de estabilidad laboral (que lograría en Berkeley en julio de 1958) le movieron a probar su capacidad en ese campo especifico ${ }^{86}$. En ese sentido, Kuhn dedicó mucho tiempo a la preparación de un primer libro de educación general en

${ }^{84}$ Con esa idea abre Koyré su Del mundo cerrado al universo infinito, loc. cit., p. 1. Para un mayor detalle puede verse su conferencia sobre "La influencia de las concepciones filosóficas en las teorías cientificas", pronunciada en Boston para la American Association for the Advancement of Science y publicada en The Scientific Monthly en 1955. En castellano puede verse la traducción de Antonio Beltrán en Koyre, Pensar la ciencia, loc. cit., cap. 1 (pp. 47-69).

${ }^{85}$ En ciencia normal (o periodo clásico) se expande una teoría; en un periodo de crisis se lucha por esa misma teoría; en una revolución científica se trabaja desde una teoría alternativa a la hasta entonces imperante. Cf. KUHN, The Quest for Physical Theory, loc. cit., conferencia 5, pp. 6-7 a 7-5. Entre los lugares de Structure donde esta idea aparece más claramente, cf. sus secciones $\mathrm{V}$ y X y los comentarios sobre las condiciones del compromiso del científico con una orientación («paradigma” en este caso) en la p. 90.

${ }^{86}$ Cf. las actas del comité de Berkeley para la promoción interna del profesorado: «BBC Minutes-November 19, 1957-BBC COMMENTS on candidates for promotion-Thomas Samuel Kuhn-HISTORY-and PHILOSOPHY 1/2 Berkeley Campus-promotion 7/1/58: Assistant Professor to Associate Professor of the History of Sciencem, Dossier de Thomas S. Kuhn, The Bancroft Library, Berkeley, California: The University of California, Berkeley, pp. 1-2. 
la línea de Conant, The Copernican Revolution. El texto apareció en 1957 a pesar de que la fecha inicial prevista por Kuhn para su finalización era septiembre de $1954^{87}$. Esto retrasó asimismo el trabajo más filosófico propio de las Conferencias Lowell. Este trabajo sólo logró aparecer en una segunda monografia, en principio bastante breve (muy por debajo del centenar de páginas), para la International Encyclopedia of Unified Science (Structure), cuya preparación se retrasó hasta el curso 1958/59 (convirtiéndola entre tanto en un libro en toda regla). En ese año Kuhn pudo al fin desvincularse de las tareas docentes. Ya fijo en su puesto de associate professor en Berkeley, pasó un año en el Center for Advanced Study in the Behavioral Sciences, en Palo Alto, California, un periodo de pura investigación que le permitió volver por sus fueros filosóficos y escribir la monografía con lo mejor de las primitivas Conferencias Lowell.

Pero durante ese largo paréntesis historiográfico Kuhn desarrolló un punto de vista propio que divergía no sólo de Conant, sino también de Koyré. Ya en las Conferencias Lowell, Kuhn había hecho uso de ejemplos históricos que se correspondían con una línea más próxima a Conant; por ejemplo, la transición de la química del flogisto a la del oxígeno como ilustración de los efectos de la orientación cientifica ${ }^{88}$. El ejemplo de la Revolución Química de A.-L. Lavoisier era tan válido para Kuhn como el de Galileo. En ambos se manifestaba un cambio en el planteamiento y objetivos de la investigación; un "cambio de problema". Los ejemplos diferían sin embargo en la manifestación histórica, particular, de ese cambio, ya que el vehículo y el modo de trabajo científico de Lavoisier y Priestley (de por sí divergente ya entre ellos dos) no guardaba prácticamente ninguna relación con el de Aristóteles o Galileo. Las Conferencias Lowell tendían, no obstante, a disminuir la distancia entre ellos, y un poco al modo unificador más propio de Koyré, Kuhn exploraba las bases psicológicas del trabajo y el cambio científico

${ }^{87}$ Para más detalles al respecto, cf. Thomas S. KUHN, "Plans of Research", 22 de octubre de 1953, Dossier de Thomas S. Kuhn, loc. cit., p. 2; y la carta de Thomas S. Kuhn a Charles W. Morris, 15 de septiembre de 1955, Thomas S. Kuhn Papers, loc. cit., pp. 1-2. Agradezco a Karl Hufbauer su manuscrito de la conferencia titulada "Kuhn's Discovery of History (1940-1958)", preparada para el simposium The Legacy of Thomas $S$. Kuhn (Cambridge [Mass.]: Dibner Institute for the History of Science and Technology, MIT, 20-22 de noviembre de 1997, sin actas publicadas), cuyo apéndice B fecha los "Plans of Research" de Kuhn.

${ }^{88}$ Cf. p. ej. KUHN, The Quest for Physical Theory loc. cit., conferencia 4, p. 5-5; ibid., conferencia 5, pp. 2a-1 y sigs. Conant se habla dedicado al ejemplo histórico en una de sus case histories: James B. ConANT, The Overthrow of the Phlogiston Theory: The Chemical Revolution of 1775-1789, Cambridge (Mass.): Harvard University Press, 1948. 
en diversos casos históricos sin más distinciones. Sin embargo, Kuhn pudo ver poco a poco a lo largo de su labor docente en historia de la ciencia que el científico está apegado a un entorno social que es el elemento causal del trabajo normal de la ciencia y de sus cambios revolucionarios (i.e., su manifestación histórica particular). Por ejemplo, la ciencia colaboró poco en el desarrollo de la Revolución Industrial en Europa pero, al contrario, el origen de la termodinámica está vinculado a ella. Los estudios sobre la eficiencia de las máquinas de vapor aportaron el vocabulario y muchos de los objetos científicos que estuvieron implicados en el desarrollo de las leyes de la termodinámica ${ }^{89}$. Kuhn daba clase en Harvard a este respecto (y en estos términos) a la par que se ocupaba de la Revolución Científica del siglo XVII ${ }^{90}$. Sin duda los elementos causales contextuales no son puros accidentes para la ciencia, sino factores de desarrollo con los que se reconstruye el escenario de trabajo de los científicos.

Cuando Kuhn se ocupa de los factores causales de naturaleza social, no se refiere a la influencia de, por ejemplo, los estados de opinión social, las ideologías, la religión o la política en el desarrollo científico. Este punto de vista, propio de estudios que en su momento (desde los ańos treinta y cuarenta del siglo pasado) se llamaron "externalistas", fue el prisma original desde el que la primera sociología de la ciencia enfocó sus trabajos ${ }^{91}$. Aunque Kuhn se adentró en el estudio de

${ }^{89}$ Kuhn trataba este episodio histórico en su seminario sobre termodinámica. Para los contenidos del seminario, cf. las notas de Kuhn para History of Science 212, 4 de febrero 195415 de noviembre de 1955. Thomas S. Kuhn Papers, loc. cit., caja 1, carpeta 22. Su artículo "Energy Conservation as an Example of Simultaneous Discovery" (en Marshall Clagett [ed.], Critical Problems in the History of Science, Madison [Wis.]: University of Wisconsin Press, 1959, pp. 321-356, reeditado en KUHN, Essential Tension, loc. cit., cap. 4 [pp. 66-104]) es resultado del trabajo junto a sus alumnos de doctorado en ese seminario.

${ }^{90}$ Cf. infra.

${ }^{91}$ La obra de Robert K. Merton es central en este enfoque. Para Merton, la sociologia de la ciencia adquiere su fundamento en la diferencia entre las condiciones internas y externas del desarrollo del conocimiento cientifico. Las primeras son las propias del método filosófico, las condiciones de avance racional del conocimiento (i.e., mediante la exploración desinteresada de los fenómenos y la construcción teórica sólo a partir de esa indagación y mediante el uso de un razonamiento lógico pulcro). Las segundas induyen todos los avatares y circunstancias de carácter moral, económico, político, etc., que afectan (y eventualmente modifican) un desarrollo que en otras circunstancias hubiera sido del todo racional. Para la perspectiva de Merton cf. su Ciencia, tecnologia y sociedad en la Inglaterra del siglo XVII, trad. N. Míguez, Madrid: Alianza, 1984 (orig. ed. rev. 1970; $1^{\text {a }}$ ed. 1938). Cf. también los diversos estudios de sociología de la ciencia recogidos en Robert K. MERTON, The Sociology of Science, Chicago: The University of Chicago Press, 1973. Sobre Merton cf. Carlos Solis, Razones e intereses: La historia de la ciencia después de Kubn, Barcelona: Paidós, 1994, pp. 65-67. 
los factores sociológicos por esa puerta en estos años (i.e., al principio de su carrera como historiador), después ayudó a corregir la idea de "factor causal del desarrollo científico de naturaleza social". Con su nueva perspectiva, esta idea supera la diferencia entre factores internos (i.e., racionales) y externos del desarrollo científico. Kuhn escribió sobre ella cuando Philipp Frank le invitó a formar parte del programa de sociología de la ciencia que el Instituto para la Unidad de la Ciencia (organismo promotor de la ya citada International Encyclopedia of Unified Science) había organizado a finales de 1952. En el borrador que se conserva de esa respuesta, Kuhn le dice a Frank:

Muchas gracias por su invitación a unirme al comité organizador del proyecto sobre "sociología de la ciencia» del instituto. No hay otro grupo en el que me gustara participar más que en éste. (...) Permítame plantear un problema sobre el borrador del prospecto adjunto. ¿No sería apropiado incluir entre los términos de referencia del comité un examen de aquellos factores sociológicos que afectan al científico individual no por ser miembro de una comunidad nacional (como los Estados Unidos) sino por ser miembro de un grupo profesional más restringido (como la American Physical Society)? Sospecho que en la ciencia occidental del siglo XX las "fes» socialmente condicionadas, implícitas, profesionales, han asumido muchos de los roles de guía de la investigación y de determinación de la aceptabilidad de las teorías científicas que las religiones y los sistemas metafísicos jugaron en las ciencias físicas del siglo XVII.

Creo que este consenso profesional tiene que ver de manera importante con los problemas que cada científico considera dignos de ataque, los experimentos que emplea para resolver sus problemas, los aspectos abstractos de sus experimentos que considera relevantes y los criterios lógicos y experimentales que exige a un argumento "válido". Soy de la opinión que un examen de tales factores en su contexto histórico arrojaría dudas sobre la posibilidad de distinguir "entre la aceptación de una teoría porque sus consecuencias concuerdan con los hechos observados, y la aceptación de una teoría a causa de otros factores.... $)^{92}$.

92 Borrador (s. f.) de una carta de Thomas S. Kuhn a Philipp G. Frank, ca. diciembre de 1952-enero de 1953, Thomas S. Kuhn Papers, loc. cit., caja 25, carpeta 53, pp. 1-2. La cita entrecomillada del propio Kuhn hace referencia al prospecto adjunto a la carta de Frank y firmado por Philipp FrANK, Robert K. MERTON y Ernest NAGEL, "Research Project in the Sociology of Science", s. f., Thomas S. Kuhn Papers, loc. cit., caja 25, carpeta 53, p. 1. Frank se puso en contacto con Kuhn en una carta del 2 de diciembre de 1952 (en Thomas S. Kuhn Papers, ibidem), a la que iba adjunto el citado prospecto. 
De este modo, al paralelismo Koyré-Piaget desarrollado por Kuhn en las Conferencias Lowell le faltaba todavía especificar el mecanismo causal. Es decir, al paralelismo en el desarrollo mente-teoría científica le faltaba un mecanismo adecuado que mostrase de dónde salen las orientaciones, cómo se generan las teorías científicas, y que nos permitiera creer en la semejanza entre el desarrollo mental individual y el de esas teorías a lo largo de su historia. Faltaba algo, en fin, que nos ayudara a admitir que la mente y la teoría eran conceptos parejos desde un prisma evolutivo ${ }^{93}$.

La labor de Kuhn como docente y como investigador de historia de la ciencia siguió esta perspectiva, es decir, para el historiador era necesario investigar qué fundamentos había en cada época para la investigación científica; qué problemas, de qué clase y de dónde provenían, y qué medios y qué criterios eran válidos para su solución. Así, por ejemplo, la investigación histórica del estudio del movimiento de Galileo a Newton y la del cambio químico en la época de Robert Boyle eran problemas a priori independientes. Y no sólo a priori. Para Kuhn, el primero había sido un problema tratado con herramientas principalmente geométricas (y juzgado con criterios también geométricos) cuyos orígenes se remontaban al periodo griego. El experimento cumplía un papel muy reducido en todo ello. Al contrario, el cambio químico bebía de fuentes que no podf́an remontarse más allá del Renacimiento. El desarrollo instrumental, el experimento controlado y el informe exacto de los resultados así obtenidos constituían el medio de

${ }^{93}$ Conviene recordar a este respecto la conexión a menudo mencionada entre los argumentos sociológicos de Kuhn y la obra del polaco Ludwik FLECK, Entstehung und Entwicklung einer wissenschaftichen Tatsache: Einfubrung in die Lehre vom Denkstil und Denkkollektiv, Frankfurt am Main: Suhrkamp Verlag, 1980 (orig. 1935). El propio Kuhn reconoció el impacto de la lectura de esa obra en sus primeros años como fellow de Harvard. En su prefacio a la traducción inglesa del libro de Fleck lo calificó como «el mejor y más importante trabajo metodológico de este siglo, hasta donde yo sé». (Thomas S. KUHN, "Foreword", en Ludwik FLECK, The Genesis and Development of a Scientific Fact, Robert K. Merton y Thaddeus J. Trenn [eds.], trad. de T. J. Trenn, Chicago: The University of Chicago Press, pp. vii-xi, esp. p. viii.) Fleck mostraba un enfoque paralelo al suyo, lo que había sido muy estimulante, pero en realidad Kuhn no extrajo demasiado de él, con la excepción de la terminología de "estilo de pensamiento" que, no obstante, rara vez (si alguna) incorporó en sus publicaciones. Y, al contrario, la idea de "colectivo de pensamientom le resultaba sumamente incómoda, ya que, como Kuhn indicaba: «Era claro que se refería a un grupo, porque era un colectivo, pero el modelo de Fleck era la mente y el individuo. Era justo lo que no me gustaba, no podfa utilizarlo. No podía meterme en ello y me parecía un tanto repugnante. Esta consideración me ayudó a mantenerme alejado de ello, pero fue muy importante que leyera el libro porque me permitió pensar: "Vale, no soy el único que ve las cosas así». BALTAS et al., op. cit., p. 330. 
comunicación y el criterio de excelencia básico. De hecho, esos dos problemas representaban para Kuhn a dos tradiciones científicas distintas, una "clásica" y la otra «baconiana", que se desarrollaron en paralelo, casi sin interacciones, hasta el final del siglo $\mathrm{XIX}^{94}$.

Tanto las clases como las publicaciones de Kuhn se distribuyeron por ambos terrenos. Como docente en Harvard, una vez que logró impartir clases más allá del curso de Conant, Kuhn se ocupó del desarrollo de la cosmología cientifica de Aristóteles a Newton ${ }^{95}$ (History of Science 105, curso 1953/54), una cuestión propia de las citadas «ciencias (matemáticas) clásicas». Y en el mismo curso (segundo cuatrimestre), Kuhn impartió un seminario sobre los orígenes de la termodinámica ${ }^{96}$. Mientras que el primer trabajo dio como fruto su The Copernican Revolution, el segundo generó la línea historiográfica más estable de Kuhn (si excluimos su trabajo sobre las fuentes de la física cuántica): la que le llevó a explorar en profundidad el trabajo de Sadi Carnot y el origen de la primera ley de la termodinámica (el principio de conservación de la energía ${ }^{97}$.

El Kuhn de mediados de los años cincuenta guardaba ya poca relación con el que vimos en las Lowell. Ahora poseía un conocimiento factual sólido acerca de la naturaleza y el origen social de las orientaciones y los objetos científicos. Y esta

${ }^{94}$ Cf. Thomas S. KUHN, «Mathematical versus Experimental Traditions in the Development of Physical Science», Journal of Interdisciplinary History, 7 (1976), pp. 1-31. Citamos por la reedición KUHN, Essential Tension, loc. cit., cap. 3 (pp. 31-65).

95 Una buena descripción de los objetivos y contenidos del curso aparece en la propuesta original que Kuhn realizó al Comité de Educación General de Harvard por vía de su presidente, David Owen. Cf. la carta de Thomas S. Kuhn a David Owen, 6 de enero de 1951, Thomas S. Kuhn Papers, loc. cit., caja 5, carpeta 84, pp. 1-2.

${ }^{96} \mathrm{Cf}$. los contenidos del seminario en las notas de Kuhn para History of Science 212, 4 de febrero 1954-15 de noviembre de 1955, loc. cit.

${ }^{97}$ Fundamentales a este respecto son los siguientes artículos de KUHN: "Carnot's Version of "Carnot's Cycle", American Journal of Physics, 23 (1955), pp. 91-95, una corrección de algunos argumentos sobre el significado del ciclo de Carnot realizados por Victor K. LA MER en sus dos artículos "Some Current Misconceptions of N. L. Sadi Carnot's Memoir and Cycle", Science, 109 (10 jun. 1949), p. 598 y "Some Current Misinterpretations of N. L. Sadi Carnot's Memoir and Cycle", American Journal of Physics, 22 (1954), pp. 20-27. La Mer contestó a Kuhn en su "Some Current Misinterpretations of N. L. Sadi Carnot's Memoir and Cycle. II", American Journal of Physics, 23 (1955), pp. 95-102, y Kuhn lo hizo a su vez en "La Mer's Version of "Carnot's Cycle", American Journal of Physics, 23 (1955), pp. 387-389. Asimismo son fundamentales en esta línea de Kuhn su "The Caloric Theory of Adiabatic Compression", Isis, 49 (2) (jun. 1958), pp. 132140, y su "Energy Conservation as an Example of Simultaneous Discovery", loc. cit. 
perspectiva más detallada lo conducía a discrepar de Koyré, en especial en lo referente a dos ideas visibles en Koyré. La primera de ellas, el carácter casi monolíico de la ciencia visible en el historiador ruso. La segunda, su negación del papel de la ciencia promulgada por F. Bacon en el desarrollo de la ciencia. Nos vamos a ocupar primero de esta segunda, y pasaremos a hablar de la primera en el apartado final del artículo.

Kuhn consideraba que Koyré extrapolaba con demasiada facilidad el modo baconiano de experimentación a cualquier periodo histórico en que ésta apareciera como aspecto central del trabajo científico. La visión de Koyré se manifestaba bien, por ejemplo, en su perspectiva sobre B. Pascal y sus experimentos sobre el vacío, que trataban de refutar la perspectiva aristotélica del horror vacui natural.

En matemáticas, Pascal era para Koyré un geómetra genial, opuesto a la corriente algebrista de Descartes, lo que le habría impedido el paso hacia los métodos de Leibniz y Newton basados en infinitesimales, pero hábil y capaz de dominar tanto el "método de los antiguos" (como Koyré denomina al tratamiento geométrico clásico) como los métodos más recientes ${ }^{98}$. Como experimentador, el Pascal de Koyré es también un hombre de gran talento. Suyo es, nada menos, el famoso experimento del Puy de Dôme, recuerda Koyré; un experimento magníficamente ejecutado ${ }^{99}$. Sin embargo, en lo que respecta a la ejecución de los experimentos, Koyré se muestra crítico. Los Nuevos experimentos concernientes al vacio de Pascal (cuyo objetivo, dice Koyré, era refutar el citado horror vacui y mostrar que los efectos que normalmente se le atribuyen están causados por el peso del aire ambiental) fueron ejecutados de un modo diferente a como él los describió en esa obra. Por ejemplo, Pascal decía haber usado tubos de vidrio de unos quince metros, para Koyré de fabricación muy difícil (quizá inviable) en la Francia del siglo XVII; una vez fabricados también habrían sido muy difíciles de manejar ${ }^{100}$. Además, había fenómenos que, para Koyré, deberían aparecer en los Nuevos experimentos, como el burbujeo del agua de los experimentos del vacío de Gasparo Berti en Roma ${ }^{101}$. Koyré no ponía en duda que Pascal hubiera hecho los experimentos, pero para él jamás informó convenientemente del resultado real, de

${ }^{98}$ Alexandre KOYRE, "Pascal como científico" (orig. 1954), en sus Estudios de historia del pensamiento cientifico, loc. cit., p. 362.

${ }^{99}$ Ibid., p. 375.

100 lbid., p. 369.

101 Ibid., p. 371. 
lo que realmente vio ${ }^{102}$. Esto, en palabras de Koyré, «(...) modifica sensiblemente la imagen tradicional de Pascal, experimentador sagaz y prudente que la convención histórica opone al apriorista impenitente que se llama Descartes. No, Pascal no es un discípulo fiel de Bacon, una primera edición de Boyle» ${ }^{103}$.

Para Kuhn, esta última frase era completamente correcta, pero no todo cuanto estaba implicada en ella. Pascal no era un alumno aventajado de Bacon previo a Robert Boyle (un fiero crítico de sus experimentos, por cierto). A decir verdad, para Kuhn, Boyle y Pascal representaban tradiciones distintas, y sólo la coincidencia puntual en el problema (fundamental) del vacío había hecho que tales modos divergentes de trabajo se encontraran.

En Pascal, decía Kuhn, los experimentos eran más bien "experiencias" y tenían un carácter demostrativo. Kuhn englobaba a Pascal en una tradición clásica, en la que un papel importante en la investigación cientifica lo ocupaba el experimento imaginario, un recurso que permite investigar la estructura del mundo dentro de - como diría Koyré- "los márgenes de lo posible», sin obtener nuevos datos pero sí nueva información. Como Kuhn señalaba en su artículo de 1964 sobre el experimento imaginario, sus efectos, «(...) aún cuando no presenta nuevos datos, se parecen más a los de la experimentación real de lo que se suele suponer. (...) Los experimentos imaginarios ayudan a que el cientifico llegue a leyes y teorías diferentes de las que antes había sostenido" ${ }^{104}$. Este uso del experimento imaginario pone de manifiesto un rasgo básico de las ciencias clásicas que Kuhn subrayaría años más tarde, en 1976, al mencionar que en ellas la exploración empírica, incluso la pulcritud de observación con el uso de instrumentos propia de la astronomía antigua y la óptica medieval, estaba sometida a la experiencia cotidiana $-\mathrm{y}$ exclusivamente a ella $-{ }^{105}$. Las formas de la experimentación inclúan pulir situaciones normales, en una observación al alcance de todos. El uso de instrumentos y recursos experimentales como el plano inclinado, el prisma, el péndulo o el barómetro servían del mismo modo que el razonamiento ló-

102 Ibid., p. 370.

${ }^{103}$ Ibid., p. 372.

104 Thomas S. KUHN, "A Function for Thought Experiments", en L'aventure de la science, Mélanges Alexandre Koyré, vol. 2, París: Hermann, 1964, pp. 307-334. Citamos por la reedición en KUHN, The Essential Tension, loc. cit., cap. 10 (pp. 241-265), p. 242.

${ }^{105}$ KUHN, «Mathematical versus Experimental Traditions in the Development of Physical Sciencem, loc. cit., p. 38. 
gico y la creatividad con que se desarrollaban los instrumentos imaginarios. Como dice A. van Helden, en esta tradición "(...) no se trataba de trascender la capacidad de discriminación de los sentidos o de ejercer violencia sobre la naturaleza". "La naturaleza", dice este historiador, "se observa sólo con los sentidos, y sólo se usan las herramientas más sencillas»" ${ }^{106}$.

En cambio, Robert Boyle sí que trabaja en una tradición baconiana. En la tradición de Bacon, el experimento y el instrumento nos lleva más allá de la situación común, a un terreno por completo desconocido a los sentidos, a la observación de (y al pensamiento sobre) lo cotidiano. La tradición baconiana, sin que sea cultivada por ingenieros y artesanos en sí, nace con el desarrollo de las artes y la instrumentación, con la aparición de la brújula, de las atracciones magnéticas, del termómetro, del telescopio, del microscopio y de la botella de Leyden. Y aquí sí que cumple un papel la situación extrema, la violencia sobre la naturaleza, el aislamiento del fenómenos y el posterior informe exhaustivo. La experiencia que se busca obtener concierne a un marco ontológico en el que prima lo desconocido ${ }^{107}$. Aquí cabría recordar el nuevo marco renacentista de Koyré mencionado más arriba, en el que la realidad se da la mano con lo posible y es un vasto terreno yermo a explorar, ya no jerarquizado como en el aristotelismo. Pero el baconianismo que $\mathrm{R}$. Boyle (entre otros) contribuye a consolidar no es exactamente la actitud del Renacimiento de la que habla Koyré. Como Kuhn señala, las orientaciones atomistas permiten una violencia sobre la naturaleza que no contribuye a la destrucción del fenómeno que se trata de observar. A diferencia de un Cosmos habitado por fuerzas ocultas, que siguen su propia voluntad, o jerarquizado como el de Aristóteles, en un universo atomista el experimento y la medición instrumental sólo intenta conocer mejor un mecanismo causal que se da tanto en las situaciones narurales como en las más artificiales ${ }^{108}$. La tradición baconiana se desarrolló poco a poco, primero explorando un terreno desconocido para alcanzar más tarde la perspectiva de búsqueda específica del detalle que había caracterizado a las ciencias clásicas en la época de la Revolución Científica del siglo XVII.

106 Albert VAN HELDEN, "The Birth of Modern Scientific Instrument, 1550-1700", en John G. Burke (ed.), The Uses of Science in the Age of Newton, Berkeley (Cal.): University of California Press, 1983, cap. 3 (pp. 49-84), esp. p. 50.

107 Cf. KUHN, «Mathematical versus Experimental Traditions in the Development of Physical Sciencen, Loc. cit., pp. 42-47.

${ }^{108} \mathrm{Ibid}$. pp. 43-44 y 54-55. Las ideas metafisicas relativas al mecanismo en sí se distinguían bien, no obstante, del trabajo y el informe puramente experimental, como Kuhn subraya (ibid, p. 44). 
Cuando esto ocurrió, las disciplinas físicas resultantes abanderaron una nueva revolución (la «segunda» de Kuhn) ${ }^{109}$.

Pese a la publicación tardía de esta distribución de Kuhn de tradiciones y funciones de los instrumentos científicos, la idea ya aparecía en ciertas críticas que éste le hizo a Koyré en 1959, con motivo de la presentación de la segunda versión de su escrito sobre la función de la medición en el desarrollo de la física. La crítica surgió con ciertos comentarios de D. J. de S. Price sobre el cambio de actitud hacia los números de Boyle que Koyré protestó. Price dijo que hasta Boyle no hay una concepción de la generalización (o ley) como proposición obtenida a partir de la enumeración de resultados no exactamente coincidentes. "Ésta, sugirió [Price]", recordaba Kuhn, "era la primera vez que conocía en que un científico se había mostrado dispuesto a proporcionar números que no se ajustan de manera precisa a una generalización simple y sin embargo extraer esa generalización simple a partir de ellos ${ }^{110}$. Koyré intervino entonces para mostrar que esto no caracterizaba a ninguna nueva actitud hacia los números que fuera clave en el desarrollo de la Revolución Científica del siglo XVII, como Price pretendía. Para Koyré, esa actitud estaba detrás de la repetición crítica de M. Mersenne de los experimentos de Galileo con el plano inclinado.

Kuhn mostró su oposición a Koyré en ese instante y para ello habló del caso de Pascal del modo siguiente:

Entonces volví a entrar en la discusión y, para mi consternación, regalé mucho material que habia esperado guardarme para otro momento. Defendí que la observación de Price era mucho mejor de lo que Koyré concedía y que, en realidad, era simplemente una manifestación más del efecto del baconianismo en la práctica de la ciencia del siglo XVII. (...) Un ejemplo muy significativo de esta transición más fundamental [hacia la tradición baconiana] la proporcionaba, sugeri, la famosa reseña de Boyle del Traité de Pascal. Ese libro está lleno de maravillosos diagramas y descripciones de experimentos. A primera vista parece un ejemplo excelente de la nueva actitud del siglo XVII.

109 Ibid., pp. 47 y sigs. Cf. también KUHN, "The Function of Measurement in Modern Physical Science», loc. cit., pp. 218-220.

110 Carta de Thomas S. Kuhn a Robert K. Merton, 7. de diciembre de 1959, Thomas S. Kuhn Papers, loc. cit., caja 23, carpeta 6, p. 4. 
Pero, como Boyle señala, no es nada de eso. A pesar de los diagramas los experimentos de Pascal son experimentos demostrativos o experimentos imaginarios de un tipo relativamente familiar en la Edad Media. Boyle señala que no era posible construir gran parte del aparato y que incluso con el aparato disponible muy pocos de los experimentos se podrían haber realizado. (...) Boyle, entonces, ejemplifica en este intercambio de ideas la novedad real de la experimentación del siglo XVII de la que la nueva actitud hacia los números de Price es parte. Esa novedad no es la creencia en que la observación y el experimento son básicos para la ciencia, porque, como Crombie ha mostrado de manera brillante, esa actitud y una filosofía metodológica que la acompañaba estaban muy desarrolladas durante la Edad Media. Lo que era novedoso era más bien la insistencia en que eran necesarios montones y montones de experimentos (la historia natural baconiana) y que se debía informar de todos los experimentos con detalle naturalista tras una ejecución real, preferiblemente en presencia de testigos. Sugerí que se podía descubrir más evidencia sobre esta transición al contrastar la actitud de Galileo hacia la experimentación, cualitativa o cuantitativa, con la de Newton. ${ }^{111}$

En suma, desde muy pronto, Kuhn se oponía a considerar que el baconianismo fuera un fraude (una expresión que él mismo asigna a Koyré) ${ }^{112}$. Para Kuhn, el programa de Francis Bacon había dado lugar a nuevas actitudes y a la búsqueda y hallazgo de nuevas experiencias. Cuando las ciencias nacidas bajo su égida alcanzaron la mayoría de edad (i.e., un estado de desarrollo teórico y especificidad descriptiva semejante a la de las ciencias clásicas en el siglo XVII), se convirtieron en la ciencia de hoy en día; la ciencia en que él mismo se había educado. La perspectiva de Koyré ayudaba a suprimir la posibilidad de comprender esas ciencias, nuestras ciencias, así como las funciones diferentes del experimento y sus variaciones como elemento causal del desarrollo científico. Kuhn sólo podía situarse en un punto de vista crítico hacia su maître en este plano. En nuestro siguiente apartado, el final, veremos en qué perspectiva general se resume esa crítica, a la par que pasamos revista a lo que hemos examinado hasta aquí.

111 Ibid., p. 5.

112 KUHN, "Mathematical versus Experimental Traditions in the Development of Physical Sciencen, loc. cit., p. 46. 


\section{Epílogo: La historia de la ciencia de Kuhn}

Nunca hubo una colaboración propiamente dicha entre Koyré y Kuhn, pero sí un gran respeto, mutua admiración $y$, a pesar de sus críticas, también agudas defensas de la historiografia de Koyré por parte de Kuhn. Por ejemplo, tras la publicación de Koyré de su From the Closed World to the Infinite Universe en 1957, James R. Newman reseñó ese texto junto a The Copernican Revolution de Kuhn, publicado el mismo año. La reseña de Newman era muy favorable para Kuhn; de hecho lo elogiaba. Pero Newman lo contrastaba precisamente con el libro de Koyré, para él poco explicativo, cargado de extractos y de notas y, en general, poco útil ${ }^{113}$. Kuhn contestó con una carta pública a los editores de la revista en la que había aparecido la reseña de Newman (Scientific American), en la que decía:

Sres.:

No puedo más que sugerir que muchos de sus lectores pueden encontrar un placer y un estímulo inmensamente mayores en el nuevo libro de Koyré (...) de lo que la reseńa de James R. Newman en su número de octubre daría a entender. (...) Mi admiración por el trabajo de Newman y la amabilidad con que ha descrito mi propio The Copernican Revolution en la misma reseña normalmente dictaría silencio. Pero ya que mi perspectiva de la historia de la ciencia debe más a Koyré que a ningún otro investigador vivo, me sorprende y me desconcierta descubrir que, mientras se ve que mi libro proporciona «evidencia alentadora de un giro renovador en el estudio de la historia de la ciencia», el de Koyré ejemplifica "la perspectiva ortodoxa» de la que Newman recomienda que se aparten a los historiadores de la ciencia. ${ }^{114}$

Koyré se sintió "profundamente conmovido" por la carta de Kuhn y le envió una breve nota de agradecimiento. I. B. Cohen le confirmó a Kuhn que la carta había emocionado a Koyré (ambos estaban juntos en Princeton el día que Koyré la leyó) $)^{115}$.

113 James R. NeWMaN, "Three Works about the Transition from Ancient to Modern Astrónomy", Scientific American, (oct. 1957), pp. 155-162. Discoveries and Opinions of Galileo de Stillman Drake era el tercer libro reseñado por Newman, con mejor suerte que el de Koyré.

${ }^{114}$ Carta de Thomas S. Kuhn a los editores de la revista, Scientific American, (dic. 1957), p. 10.

115 Carta de Alexandre Koyré a Thomas S. Kuhn, 22 de diciembre de 1957; carta de I. Bernard Cohen a Thomas S. Kuhn, 12 de diciembre de 1957; ambas en Thomas S. Kuhn Papers, loc. cit., caja 25, carpeta 49. 
A pesar de la fiera defensa kuhniana de su padre intelectual, Newman posiblemente estaba poniendo el dedo en la llaga más dolorosa. La historia de la ciencia de Kuhn guardaba ya poca relación con la de Koyré. Structure, de hecho, sería reconocida por el propio Koyré como una obra uinteresante» y «excitante». El concepto de paradigma, decía Koyré, junto a «las consideraciones socio-psicológicas sobre la conducta de la "profesión" le parecían "realmente iluminadoras" a la par que "llenaban el hueco entre la historia de la ciencia como tal y la historia social que hasta ahora estaban a kilómetros de distancia” ${ }^{16}$. Pero lo cierto era que Koyré trabajaba en el seno de esa misma perspectiva en la que el internalismo y el externalismo eran dos conceptos con completo sentido. Para Koyré, como para Merton por ejemplo, habia una columna vertebral de toda ciencia constituida exclusivamente por las ideas y su manejo. Aunque los elementos provenientes del contexto social, tecnológico, económico, etc., afectaran a esa médula espinal, no formaban parte de ella. Kuhn hablaría sobre ello y de este mismo modo años después, cuando dijera que, al igual que otros historiadores, “(...) Alexandre Koyré no [había] dudado en atribuir un papel importante en el desarrollo científico a las ideas extracientíficas (...) [y se había] resistido ante todo a la atención a los factores socioeconómicos e institucionales tal como los trataban B. Hessen, G. N. Clark y R. K. Merton" ${ }^{117}$. Koyré, por así decirlo, habían ampliado un círculo destinado exclusivamente a los argumentos y su origen, pero en realidad no había anulado la línea de división que separaba a la ciencia del medio social en que se desarrollaba.

Kuhn sí dio ese paso, y Koyré se lo reconoció con las frases arriba reproducidas. Kuhn recordaba en los años ochenta que una de sus principales preocupaciones al escribir Structure había sido no olvidarse del medio social como factor causal del desarrollo científico. Structure, decía en 1983, le parecía exclusivamente internalista, pero la carta de Koyré le abrió los ojos: «(...) de pronto me di cuenta de lo que Koyré tenía en mente. Al subrayar que el conocimiento científico era producto de comunidades científicas establecidas en una sociedad más amplia, había descubierto una junción a través de la cual las influencias socioeconómicas que él había rechazado en otro lugar podían jugar un papel en el desarrollo de la ciencia» ${ }^{18}$. Con

116 Entrecomillados extraídos de la carta de Alexandre Koyré a Thomas S. Kuhn, reproducida por Kuhn parcialmente en sus «Reflections on Receiving the John Desmond Bernal Award", $4 S$ Review, 1 (4) (inv. 1983), pp. 26-30, esp. p. 27.

117 KUHN, "Mathematical versus Experimental Traditions in the Development of Physical Sciencen, loc. cit., p. 32, nota 1.

${ }^{18}$ KUHN, "Reflections on Receiving the John Desmond Bernal Award», loc. cit., p. 27. 
Kuhn y Structure, el círculo entorno a las ideas cientificas se volvía borroso, porque las ideas no se desarrollaban en el vacío y sin la voluntad de algún agente. No le ocurría al desarrollo individual, como ya mostraran Piaget y otros psicólogos, y tampoco al producto de muchas mentes organizadas: la teoría cientifica.

Las comunidades cientificas eran el producto a aislar en la perspectiva de Kuhn. Había que aprender su vocabulario, conocer sus recursos técnicos disponibles (con sus limitaciones) y poder dar sentido a la solución especifica de problemas por parte del científico o grupo de ellos estudiados. Para Kuhn, la historia de la ciencia no era sólo la expresión del espíritu humano en una línea de desarrollo exclusivamente intelectual, sino la suma continuamente adaptada de los productos de la investigación de muchas comunidades $\mathrm{y}$, por lo tanto, muchas ciencias de muy diversa factura. Pocas de ellas habían llegado a nosotros vivas, a pesar que nuestras ciencias fisicas, por ejemplo, se pudieran llamar con justicia herederas de Galileo, Newton, Laplace, Fourier o Kelvin. La historia de la ciencia de Kuhn era, a diferencia de la de Koyré, una estructura compleja bien arraigada en la sociedad pero algo separada de ella. Un científico contemporáneo, como Kuhn le indicaba a Frank, a Merton y a los positivistas lógicos, se caracterizaba antes y mejor por su pertenencia a las sociedades científicas que a la sociedad de su país. Así, en Structure, Kuhn mostró cómo podríamos construir un mapa sincrónico de la distribución de las disciplinas cientificas (i.e., la obra de las comunidades cientificas) a lo largo de los siglos mediante unas pautas referentes a la organización del conocimiento en las comunidades cientificas y su estructura social común. Las revoluciones marcaban el inicio de unas y el final de otras. Con ello, Kuhn invitaba a contemplar la historia de la ciencia en la flor de su complejidad natural (añadiendo, eso sí, una pizca de evolucionismo a la imagen general). Podemos calificar la nueva imagen de Kuhn con un nombre muy apropiado que él mismo aplicó exclusivamente a la ciencia en su periodo clásico (o ciencia normal): la historia de la ciencia se revela como una "estructura destartalada" (a ramshackle structure) ${ }^{119}$.

En esta transición de Kuhn, sus concepciones sobre la naturaleza de la ciencia se ponen de manifiesto en los diferentes papeles que asigna a la experimentación controlada y a los instrumentos científicos. La propia idea de una función cambiante muestra la divergencia tanto de Koyré, quien sólo vefa un papel uniforme y dependiente del trabajo con la estructura conceptual y la teoría, como de Conant

${ }^{119}$ KUHN, The Structure of Scientific Revolutions, loc. cit., p. 49. 
y Bridgman, para quienes el experimento controlado era una quintaesencia de la ciencia a la que servía todo el trabajo intelectual del cientifico. Estos tres pensadores coincidían en algo, la raíz de la ciencia estaba en el pensamiento humano, pero ninguno de ellos podía traspasar la barrera de sus principios, bien empiristas, como en el caso de Conant y Bridgman, bien neo-kantianos (e incluso fenomenológicos) en el de Koyré. Sólo Kuhn pudo hacerlo en medio de un difícil proceso de ubicación profesional y a lo largo de años de oscilación de uno a otro polo, con la sola guía de sus propias intuiciones filosóficas. Este artículo ha tratado de aislar en lo posible esa transición y el principal objeto teórico desarrollado, la nueva versión kuhniana de la función de los instrumentos y los experimentos en el desarrollo histórico de las ciencias fisicas, tratando de mostrar asimismo los puntos de inflexión del desarrollo intelectual de Kuhn acerca de esta cuestión en particular. Nuestra intención ha sido mostrar cómo Kuhn sometió a una duda más que razonable una convicción ya añeja sobre la posibilidad de la observación sin la intervención de categorias no-kantianas sin la necesidad de desvincular la ciencia de sus raices en la experiencia humana cambiante. Esperamos haber expuesto, al menos, que esta conjunción era para Kuhn la vía adecuada para la construcción del método. 